Pacific

Journal of

Mathematics

\title{
ON STABLE COMMUTATOR LENGTH IN HYPERELLIPTIC MAPPING CLASS GROUPS
}

Danny Calegari, NaOyuki Monden and Masatoshi Sato 


\title{
ON STABLE COMMUTATOR LENGTH IN HYPERELLIPTIC MAPPING CLASS GROUPS
}

\author{
Danny Calegari, NaOyuki Monden and Masatoshi Sato
}

\begin{abstract}
We give a new upper bound on the stable commutator length of Dehn twists in hyperelliptic mapping class groups and determine the stable commutator length of some elements. We also calculate values and the defects of homogeneous quasimorphisms derived from $\omega$-signatures and show that they are linearly independent in the mapping class groups of pointed 2-spheres when the number of points is small.
\end{abstract}

\section{Introduction}

The aim of this paper is to investigate stable commutator length in hyperelliptic mapping class groups and in mapping class groups of pointed 2-spheres. Given a group $G$ and an element $x \in[G, G]$, the commutator length of $x$, denoted by $\operatorname{cl}_{G}(x)$, is the smallest number of commutators in $G$ whose product is $x$, and the stable commutator length of $x$ is defined by the limit $\operatorname{scl}_{G}(x):=\lim _{n \rightarrow \infty} \operatorname{cl}_{G}\left(x^{n}\right) / n$ (see Definition 2.1 for details).

We investigate stable commutator length in two groups, $\mathcal{M}_{0}^{m}$ and $\mathscr{H}_{g}$. Let $m$ be a positive integer greater than 3. Choose $m$ distinct points $\left\{q_{i}\right\}_{i=1}^{m}$ in a 2-sphere $S^{2}$. Let Diff $+\left(S^{2},\left\{q_{i}\right\}_{i=1}^{m}\right)$ denote the set of all orientation-preserving diffeomorphisms in $S^{2}$ which preserve $\left\{q_{i}\right\}_{i=1}^{m}$ setwise with the $C^{\infty}$-topology. We define the mapping class group of the $m$-pointed 2-sphere by $\mathcal{M}_{0}^{m}=\pi_{0} \operatorname{Diff}_{+}\left(S^{2},\left\{q_{i}\right\}_{i=1}^{m}\right)$. Let $\Sigma_{g}$ be a closed connected oriented surface of genus $g \geq 1$. An involution $\iota: \Sigma_{g} \rightarrow \Sigma_{g}$ defined as in Figure 1 is called the hyperelliptic involution.

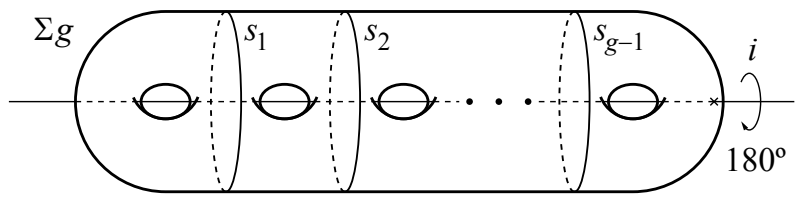

Figure 1. Hyperelliptic involution $\iota$ and the curves $s_{1}, \ldots, s_{g-1}$.

MSC2010: primary 57M07; secondary 20F12, 57N05.

Keywords: stable commutator length, mapping class groups. 
Let $M_{g}$ denote the mapping class group of $\Sigma_{g}$, that is, the group of isotopy classes of orientation-preserving diffeomorphisms of $\Sigma_{g}$, and let $\mathscr{H}_{g}$ be the centralizer of the isotopy class of a hyperelliptic involution in $\mathcal{M}_{g}$, which is called the hyperelliptic mapping class group of genus $g$. Note that $\mu_{g}=\mathscr{H}_{g}$ when $g=1,2$. Since there exists a surjective homomorphism $\mathscr{P}: \mathscr{H}_{g} \rightarrow \mathcal{M}_{0}^{2 g+2}$ with finite kernel (see Lemma 3.3 and the paragraph before Remark 3.7), these two groups have the same stable commutator length.

Let $s_{0}$ be a nonseparating curve on $\Sigma_{g}$ satisfying $\iota\left(s_{0}\right)=s_{0}$, and let $s_{h}$ be a separating curve in Figure 1 for $h=1, \ldots, g-1$. We denote by $t_{s_{j}}$ the Dehn twist about $s_{j}$ for $j=0,1, \ldots, g-1$. In general, it is difficult to compute stable commutator length, but those of some mapping classes are known. In the mapping class group of a compact oriented surface with connected boundary, Baykur, Korkmaz and the second author [Baykur et al. 2013] determined the commutator length of the Dehn twist about a boundary curve. In the mapping class group of a closed oriented surface, interesting lower bounds on scl of Dehn twists are obtained using gauge theory. Endo and Kotschick [2001], and Korkmaz [2004] proved that $1 /(18 g-6) \leq \operatorname{scl}_{\mathcal{M}_{g}}\left(t_{s_{j}}\right)$ for $j=0,1, \ldots, g-1$. For technical reasons, this result is stated in [Endo and Kotschick 2001] only for separating curves. This technical assumption is removed in [Korkmaz 2004]. The second author [Monden 2012] also showed that $1 /(8 g+4) \leq \operatorname{scl}_{\mathscr{H}_{g}}\left(t_{s_{0}}\right)$ and

$$
\frac{h(g-h)}{g(2 g+1)} \leq \operatorname{scl}_{\mathscr{H}_{g}}\left(t_{s_{h}}\right) \quad \text { for } h=1, \ldots, g-1 .
$$

Stable commutator length on a group is closely related to functions on the group called homogeneous quasimorphisms through Bavard's duality theorem. Homogeneous quasimorphisms are homomorphisms up to bounded error called the defect (see Definition 2.2 for details). By Bavard's theorem, if we obtain a homogeneous quasimorphism on the group and calculate its defect, we also obtain a lower bound on stable commutator length. Actually, Bestvina and Fujiwara [2002, Theorem 12] proved that the spaces of homogeneous quasimorphisms on $\mu_{g}$ and $\mu_{0}^{m}$ are infinite-dimensional when $g \geq 2$ and $m \geq 5$, respectively. However it is hard to compute explicit values of these quasimorphisms and their defects. To compute stable commutator length, we consider computable quasimorphisms derived from $\omega$-signature in [Gambaudo and Ghys 2005] on symmetric mapping class groups.

In Section 3, we review symmetric mapping class groups, which are defined by Birman and Hilden as generalizations of hyperelliptic mapping class groups. We reconsider cobounding functions of $\omega$-signatures as a series of quasimorphisms $\phi_{m, j}$ on a symmetric mapping class group $\pi_{0} C_{g}(t)$. Since there exists a surjective homomorphism $\mathscr{P}: \pi_{0} C_{g}(t) \rightarrow \mathcal{M}_{0}^{m}$ with finite kernel, the homogenizations $\bar{\phi}_{m, j}$ 
induce homogeneous quasimorphisms on $\mathcal{M}_{0}^{m}$. Let $\sigma_{i} \in \mathcal{M}_{0}^{m}$ be a half twist which permutes the $i$-th point and the $(i+1)$-th point. We denote by $\tilde{\sigma}_{i} \in \pi_{0} C_{g}(t)$ a lift of $\sigma_{i}$, which will be defined on page 333 .

In Section 6, we calculate $\phi_{m, j}$ and their homogenizations $\bar{\phi}_{m, j}$.

Theorem 1.1. Let $r$ be an integer such that $2 \leq r \leq m$. Then:

$$
\phi_{m, j}\left(\tilde{\sigma}_{1} \cdots \tilde{\sigma}_{r-1}\right)=\frac{2(r-1) j(m-j)}{m(m-1)} .
$$

(ii) $\bar{\phi}_{m, j}\left(\sigma_{1} \cdots \sigma_{r-1}\right)=-\frac{2}{r}\left\{\frac{j r(m-j)(m-r)}{m^{2}(m-1)}+\left(\frac{r j}{m}-\left[\frac{r j}{m}\right]-\frac{1}{2}\right)^{2}-\frac{1}{4}\right\}$,

where $[x]$ denotes the greatest integer $\leq x$.

Since this requires straightforward and lengthy calculations to prove, we leave it until the last section. A computer calculation shows that the $([m / 2]-1) \times([m / 2]-1)$ matrix whose $(i, j)$-entry is $\bar{\phi}_{m, j+1}\left(\sigma_{1} \cdots \sigma_{i}\right)$ is nonsingular when $4 \leq m \leq 30$. Thus we have:

Corollary 1.2. The set $\left\{\bar{\phi}_{m, j}\right\}_{j=2}^{[m / 2]}$ is linearly independent when $4 \leq m \leq 30$.

In Section 4, we calculate the defects of the homogenizations of these quasimorphisms. In particular, we determine the defect of $\bar{\phi}_{m, m / 2}$ when $m$ is even. Actually $\bar{\phi}_{m, m / 2}$ is the same as the homogenization of the Meyer function on the hyperelliptic mapping class group $\mathscr{H}_{g}$.

Theorem 1.3. Let $D\left(\phi_{m, j}\right)$ and $D\left(\bar{\phi}_{m, j}\right)$ be the defects of the quasimorphisms $\phi_{m, j}$ and $\bar{\phi}_{m, j}$, respectively.

(i) For $j=1,2, \ldots,[m / 2]$,

$$
D\left(\bar{\phi}_{m, j}\right) \leq D\left(\phi_{m, j}\right) \leq m-2 .
$$

(ii) When $m$ is even and $j=m / 2$,

$$
D\left(\bar{\phi}_{m, m / 2}\right)=m-2 \text {. }
$$

Remark 1.4. If $\phi: G \rightarrow \mathbb{R}$ is a quasimorphism and $\bar{\phi}: G \rightarrow \mathbb{R}$ is its homogenization, they satisfy

$$
D(\bar{\phi}) \leq 2 D(\phi)
$$

(see [Calegari 2009] Corollary 2.59). We will claim in Lemma 4.1 that, when $\phi$ is antisymmetric and a class function, they satisfy the sharper inequality

$$
D(\bar{\phi}) \leq D(\phi) .
$$

Note that when $g=2$, the hyperelliptic mapping class group $\mathscr{H}_{2}$ coincides with $\mu_{2}$. We may think of the lift of $\sigma_{i} \in M_{0}^{6}$ for $i=1,2,3,4,5$ to $\mathcal{M}_{2}$ as the Dehn twist $t_{c_{i}}$ along the simple closed curve $c_{i}$ in Figure 2 (see page 333). Similarly 
the Dehn twist $t_{s_{1}} \in M_{2}$ can be considered as a lift of $\left(\sigma_{1} \sigma_{2}\right)^{6} \in \mathcal{M}_{0}^{6}$ by the chain relation (see Lemma 2.8). Since Theorem 1.1(ii) implies $\bar{\phi}_{6,2}\left(\left(\sigma_{1} \sigma_{2}\right)^{6}\right)=-8 / 5$ and Theorem 1.3(i) implies $D\left(\bar{\phi}_{6,2}\right) \leq 4$, by applying Bavard's duality theorem, we have:

\section{Corollary 1.5.}

$$
\frac{1}{5} \leq \operatorname{scl}_{\mathcal{M}_{2}}\left(t_{s_{1}}\right) .
$$

To the best of our knowledge, for $g \geq 2$, there is not known an element $x$ in $\mathscr{H}_{g}$ (or $\mathcal{M}_{g}$ ) such that $\operatorname{scl}(x)$ is nonzero and can be computed explicitly. By Theorem 1.3(ii), we can determine the stable commutator length of the following element in $\mathcal{H}_{g}$.

Theorem 1.6. Let $d_{2}^{+}, d_{2}^{-}, \ldots, d_{g-1}^{+}, d_{g-1}^{-}$be simple closed curves in Figure 7 . Let $c$ be a nonseparating simple closed curve satisfying $\iota(c)=c$ which is disjoint from $d_{i}^{+}, d_{i}^{-}$and $s_{h}(i=1, \ldots, g, h=1, \ldots, g-1)$. For $g \geq 2$,

$$
\operatorname{scl}_{\mathscr{H}_{g}}\left(t_{c}^{2 g+8}\left(t_{d_{2}^{+}} t_{d_{2}^{-}} \cdots t_{d_{g-1}^{+}} t_{d_{g-1}^{-}}\right)^{2}\left(t_{s_{1}} \cdots t_{s_{g-1}}\right)^{-1}\right)=\frac{1}{2} \text {. }
$$

In particular, if $g=2$, then we have $\operatorname{scl}_{\mathscr{H}_{2}}\left(t_{c}^{12} t_{s_{1}}^{-1}\right)=1 / 2$.

Next we consider upper bounds on stable commutator length. Korkmaz [2004] also gave the upper bound $\operatorname{scl}_{\mathcal{M}_{g}}\left(t_{s_{0}}\right) \leq 3 / 20$ for $g \geq 2$. In the case of $g=2$, the second author [Monden 2012] showed $\operatorname{scl}_{\mathcal{M}_{2}}\left(t_{s_{0}}\right)<\operatorname{scl}_{\mathcal{M}_{2}}\left(t_{s_{1}}\right)$. However these upper bounds do not depend on $g$. On the other hand, Kotschick [2008] proved that there is an estimate $\operatorname{scl}_{\mathcal{M}_{g}}\left(t_{s_{0}}\right)=O(1 / g)$ by using the so-called "Munchhausen trick".

In Section 5, we give the following upper bounds.

Theorem 1.7. Let $s_{0}$ be a nonseparating curve on $\Sigma_{g}$, and let $G_{g}$ be either $M_{g}$ or $\mathscr{H}_{g}$. For all $g \geq 1$, we have

$$
\operatorname{scl}_{G_{g}}\left(t_{s_{0}}\right) \leq \frac{1}{2\{2 g+3+(1 / g)\}} .
$$

\section{Preliminaries}

Stable commutator lengths and quasimorphisms. Let $G$ denote a group, and let $[G, G]$ denote the commutator subgroup, which is the subgroup of $G$ generated by all commutators $[x, y]=x y x^{-1} y^{-1}$ for $x, y \in G$.

Definition 2.1. For $x \in[G, G]$, the commutator length $\mathrm{cl}_{G}(x)$ of $x$ is the least number of commutators in $G$ whose product is equal to $x$. The stable commutator length of $x$, denoted $\operatorname{scl}(x)$, is the limit

$$
\operatorname{scl}_{G}(x)=\lim _{n \rightarrow \infty} \frac{\operatorname{cl}_{G}\left(x^{n}\right)}{n} .
$$


For each fixed $x$, the function $n \mapsto \operatorname{cl}_{G}\left(x^{n}\right)$ is nonnegative and

$$
\operatorname{cl}_{G}\left(x^{m+n}\right) \leq \operatorname{cl}_{G}\left(x^{m}\right)+\operatorname{cl}_{G}\left(x^{n}\right) .
$$

Hence this limit exists. If $x$ is not in $[G, G]$ but has a power $x^{m}$ which is, define $\operatorname{scl}_{G}(x)=\operatorname{scl}_{G}\left(x^{m}\right) / m$. We also define $\operatorname{scl}_{G}(x)=\infty$ if no power of $x$ is contained in $[G, G]$ (we refer the reader to [Calegari 2009] for the details of the theory of the stable commutator length).

Definition 2.2. A quasimorphism is a function $\phi: G \rightarrow \mathbb{R}$ for which there is a least constant $D(\phi) \geq 0$ such that

$$
|\phi(x y)-\phi(x)-\phi(y)| \leq D(\phi),
$$

for all $x, y \in G$. We call $D(\phi)$ the defect of $\phi$. A quasimorphism is homogeneous if it satisfies the additional property $\phi\left(x^{n}\right)=n \phi(x)$ for all $x \in G$ and $n \in \mathbb{Z}$.

We recall the following basic facts. Let $\phi$ be a quasimorphism on $G$. For each $x \in G$, define

$$
\bar{\phi}(a):=\lim _{n \rightarrow \infty} \frac{\phi\left(x^{n}\right)}{n} .
$$

The limit exists and defines a homogeneous quasimorphism. Homogeneous quasimorphisms have the following properties, shown for example in [Calegari 2009, Section 5.5.2] and [Kotschick 2008, Lemma 2.1(1)].

Lemma 2.3. Let $\phi$ be a homogeneous quasimorphism on $G$. For all $x, y \in G$,

(i) $\phi(x)=\phi\left(y x y^{-1}\right)$,

(ii) $x y=y x \Longrightarrow \phi(x y)=\phi(x)+\phi(y)$.

Theorem 2.4 (Bavard's duality theorem [1991]). Let $Q$ be the set of homogeneous quasimorphisms on $G$ with positive defects. For any $x \in[G, G]$, we have

$$
\operatorname{scl}_{G}(x)=\sup _{\phi \in Q} \frac{|\phi(x)|}{2 D(\phi)} .
$$

Mapping class groups. For $g \geq 1$, the abelianizations of the mapping class group $\mu_{g}$ of the surface $\Sigma_{g}$ and its subgroup $\mathscr{H}_{g}$ are finite (see [Powell 1978]). Therefore all elements of $\mathcal{M}_{g}$ and $\mathscr{H}_{g}$ have powers that are products of commutators. Dehn showed that the mapping class group $M_{g}$ is generated by Dehn twists along nonseparating simple closed curves. We review some relations between them. Hereafter we do not distinguish a simple closed curve in $\Sigma_{g}$ and its isotopy class. The following relations are well known. See, for example, [Farb and Margalit 2012, Sections 3.3, 3.5.1, 5.1.4, and 4.4.1]. 


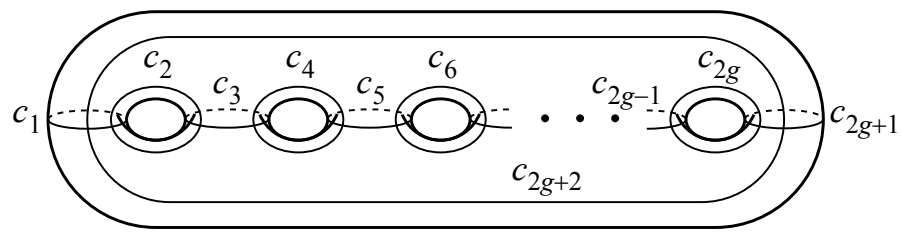

Figure 2. The curves $c_{1}, c_{2}, \ldots, c_{2 g+2}$.

Lemma 2.5. Let $c$ be a simple closed curve in $\Sigma_{g}$ and $f \in \mathcal{M}_{g}$. Then we have

$$
t_{f(c)}=f t_{c} f^{-1} \text {. }
$$

From this lemma, the values of scl and homogeneous quasimorphisms on the Dehn twists about nonseparating simple closed curves are constant.

Lemma 2.6. Let $c$ and $d$ be simple closed curves in $\Sigma_{g}$.

(i) If $c$ is disjoint from $d$, then $t_{c} t_{d}=t_{d} t_{c}$.

(ii) If $c$ intersects $d$ in one point transversely, then $t_{c} t_{d} t_{c}=t_{d} t_{c} t_{d}$.

Lemma 2.7 (hyperelliptic involution). Let $c_{1}, \ldots, c_{2 g+1}$ be nonseparating curves in $\Sigma_{g}$ as in Figure 2. We call the product

$$
\iota:=t_{c_{2 g+1}} t_{c_{2 g}} \cdots t_{c_{2}} t_{c_{1}} t_{c_{1}} t_{c_{2}} \cdots t_{c_{2 g}} t_{c_{2 g}+1}
$$

the hyperelliptic involution. For $g=1$, the hyperelliptic involution เ equals $t_{c_{1}} t_{c_{2}} t_{c_{1}} t_{c_{1}} t_{c_{2}} t_{c_{1}}$, where $c_{1}$ and $c_{2}$ are respectively the meridian and longitude of $\Sigma_{1}$.

Lemma 2.8 (chain relation). For a positive integer $n$, let $a_{1}, a_{2}, \ldots, a_{n}$ be a sequence of simple closed curves in $\Sigma_{g}$ such that $a_{i}$ and $a_{j}$ are disjoint if $|i-j| \geq 2$ and $a_{i}$ and $a_{i+1}$ intersect at one point.

When $n$ is odd, a regular neighborhood of $a_{1} \cup a_{2} \cup \cdots \cup a_{n}$ is a subsurface of genus $(n-1) / 2$ with two boundary components, denoted by $d_{1}$ and $d_{2}$. Then

$$
\left(t_{a_{n}} \cdots t_{a_{2}} t_{a_{1}}\right)^{n+1}=t_{d_{1}} t_{d_{2}} .
$$

When $n$ is even, a regular neighborhood of $a_{1} \cup a_{2} \cup \cdots \cup a_{n}$ is a subsurface of genus $n / 2$ with connected boundary, denoted by $d$. Then

$$
\left(t_{a_{n}} \cdots t_{a_{2}} t_{a_{1}}\right)^{2(n+1)}=t_{d} .
$$

Meyer's signature cocycle. Let $X$ be a compact oriented $(4 n+2)$-manifold for nonnegative integer $n$, and let $\Gamma$ be a local system on $X$ such that $\Gamma(x)$ is a finite-dimensional real or complex vector space for $x \in X$. If we are given a regular antisymmetric (respectively, skew-hermitian) form $\Gamma \otimes \Gamma \rightarrow \mathbb{R}$ (respectively, $\Gamma \otimes \Gamma \rightarrow \mathbb{C}$ ), we have a symmetric (respectively, hermitian) form on $H_{2 n+1}(X ; \Gamma)$ 
as in [Meyer 1972, p. 12]. For simplicity, we only explain the complex case. It is defined by

$$
\begin{aligned}
H_{2 n+1}(X ; \Gamma) \otimes H_{2 n+1}(X ; \Gamma) & \cong H^{2 n+1}(X, \partial X ; \Gamma) \otimes H^{2 n+1}(X, \partial X ; \Gamma) \\
& \stackrel{\cup}{\rightarrow} H^{4 n+2}(X, \partial X ; \Gamma \otimes \Gamma) \\
& \rightarrow H^{4 n+2}(X, \partial X ; \mathbb{C}) \\
& \stackrel{[X, \partial X]}{\longrightarrow} \mathbb{C},
\end{aligned}
$$

where the first row is defined by the Poincare duality, the second row is defined by the cup product of the base space, the third row comes from the skew-hermitian form of $\Gamma$ as above, and the fourth row is the evaluation by the fundamental class of $X$. Meyer showed additivity of signatures with respect to this hermitian form (more strongly, he showed Wall's nonadditivity formula for $G$-signatures of homology groups with local coefficients).

Theorem 2.9 [Meyer 1972, Satz I.3.2]. Let $X$ and $\Gamma$ be as above. Assume that $X$ is obtained by gluing two compact oriented $(4 n+2)$-manifold $X_{-}$and $X_{+}$along some boundary components.

Then we have

$$
\operatorname{Sign}\left(H_{2 n+1}(X ; \Gamma)\right)=\operatorname{Sign}\left(H_{2 n+1}\left(X_{-} ;\left.\Gamma\right|_{X_{-}}\right)\right)+\operatorname{Sign}\left(H_{2 n+1}\left(X_{+} ;\left.\Gamma\right|_{X_{+}}\right)\right) .
$$

Consider the case when $X$ is a pair of pants, which we denote by $P$. Let $\alpha$ and $\beta$ be loops in $P$ as in Figure 3, left.

For $\varphi, \psi \in \mathcal{M}_{g}$, there exists a $\Sigma_{g}$-bundle $E_{\varphi, \psi}$ on $P$ whose monodromies along $\alpha$ and $\beta$ are $\varphi$ and $\psi$, respectively. This is unique up to bundle isomorphism. In this setting, the intersection form on the local system $H_{1}\left(\Sigma_{g} ; \mathbb{R}\right)$ induces the symmetric form on $H_{1}\left(P ; H_{1}\left(\Sigma_{g} ; \mathbb{R}\right)\right)$. Meyer showed that the signature of this symmetric form on $H_{1}\left(P ; H_{1}\left(\Sigma_{g} ; \mathbb{R}\right)\right)$ coincides with that of $E_{\varphi, \psi}$. Moreover he explicitly described it in terms of the action of the mapping class group on $H_{1}\left(\Sigma_{g} ; \mathbb{R}\right)$ as
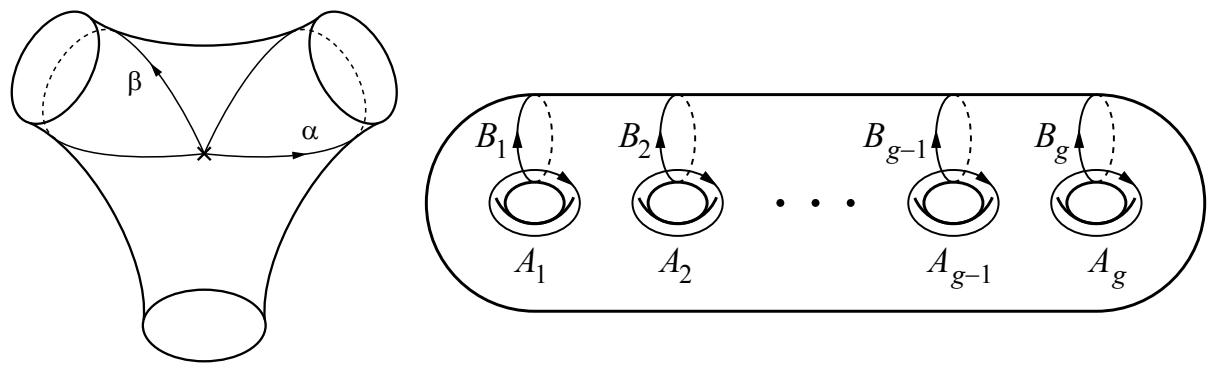

Figure 3. Left: loops in a pair of pants. Right: a symplectic basis of $H_{1}\left(\Sigma_{g} ; \mathbb{Z}\right)$. 
follows. Fix the symplectic basis $\left\{A_{i}, B_{i}\right\}_{i=1}^{g}$ of $H_{1}\left(\Sigma_{g} ; \mathbb{Z}\right)$ as in Figure 3, right; then the action induces a homomorphism $\rho: M_{g} \rightarrow \operatorname{Sp}(2 g ; \mathbb{Z})$. Let $I$ denote the identity matrix of rank $g$ and define

$$
J=\left(\begin{array}{rr}
0 & I \\
-I & 0
\end{array}\right) .
$$

For symplectic matrices $A$ and $B$ of rank $2 g$, define the vector space

$$
V_{A, B}=\left\{(v, w) \in \mathbb{R}^{2 g} \times \mathbb{R}^{2 g} \mid\left(A^{-1}-I\right) v+(B-I) w=0\right\} .
$$

Consider the symmetric bilinear form

$$
\langle,\rangle_{A, B}: V_{A, B} \times V_{A, B} \rightarrow \mathbb{R}
$$

on $V_{A, B}$ defined by

$$
\left\langle\left(v_{1}, w_{1}\right),\left(v_{2}, w_{2}\right)\right\rangle_{A, B}:=\left(v_{1}+w_{1}\right)^{T} J(I-B) w_{2} .
$$

Then, the space $V_{A, B}$ coincides with $H_{1}\left(P ; H_{1}\left(\Sigma_{g} ; \mathbb{R}\right)\right)$, and the above form $\langle,\rangle_{\rho(\varphi), \rho(\psi)}$ corresponds to the symmetric form on $H_{1}\left(P ; H_{1}\left(\Sigma_{g} ; \mathbb{R}\right)\right)$.

Meyer's signature cocycle $\tau_{g}: M_{g} \times M_{g} \rightarrow \mathbb{Z}$ is the map defined by

$$
(\varphi, \psi) \mapsto \operatorname{Sign}\left(\langle,\rangle_{\rho(\varphi), \rho(\psi)}\right),
$$

which is known to be a bounded 2-cocycle by Theorem 2.9. When we restrict it to the hyperelliptic mapping class group $\mathscr{H}_{g}$, it represents the trivial cohomology class in $H^{2}\left(\mathscr{H}_{g} ; \mathbb{Q}\right)$. Since the first homology $H_{1}\left(\mathscr{H}_{g} ; \mathbb{Q}\right)$ is trivial, the cobounding function $\phi_{g}: \mathscr{H}_{g} \rightarrow \mathbb{Q}$ of $\tau_{g}$ is unique. It is a quasimorphism, called the Meyer function. Endo [2000] computed it to investigate signatures of fibered 4-manifolds called hyperelliptic Lefschetz fibrations. Morifuji [2003] relates it to the eta invariants of mapping tori and the Casson invariants of integral homology 3-spheres.

\section{Cobounding functions of the Meyer's signature cocycles on symmetric mapping class groups}

As in the introduction, let $m$ be a positive integer greater than 3 and $\left\{q_{i}\right\}_{i=1}^{m}$ be $m$ distinct points in a 2-sphere $S^{2}$. Choose a base point $* \in S^{2}-\left\{q_{i}\right\}_{i=1}^{m}$, and denote by $\alpha_{i} \in \pi_{1}\left(S^{2}-\left\{q_{i}\right\}_{i=1}^{m}, *\right)$ a loop which rounds the point $q_{i}$ clockwise as in Figure 4.

For an integer $d$ such that $d \geq 2$ and $d \mid m$, define a homomorphism

$$
\pi_{1}\left(S^{2}-\left\{q_{i}\right\}_{i=1}^{m}\right) \rightarrow \mathbb{Z} / d \mathbb{Z}
$$

by mapping each generator $\alpha_{i}$ to $1 \in \mathbb{Z} / d \mathbb{Z}$. This homomorphism induces a $d$-cyclic branched covering $p_{d}: \Sigma_{h} \rightarrow S^{2}$ with $m$ branched points, where $\Sigma_{h}$ is a closed oriented surface of genus $h$. Applying the Riemann-Hurwitz formula, we have 
$h=(d-1)(m-2) / 2$. We denote by $t: \Sigma_{h} \rightarrow \Sigma_{h}$ the deck transformation which corresponds to the generator $1 \in \mathbb{Z} / d \mathbb{Z}$.

Let $\eta$ denote the $d$-th root of unity $\exp (2 \pi \boldsymbol{i} / d)$, where $\boldsymbol{i}$ is a square root of -1 . The first homology $H_{1}\left(\Sigma_{h} ; \mathbb{C}\right)$ decomposes into a direct sum $\bigoplus_{j=1}^{d-1} V^{\eta^{j}}$, where $V^{z}$ is an eigenspace whose eigenvalue is $z \in \mathbb{C}$. Note that $V^{1}$ is trivial since the quotient space $\Sigma_{g} /\langle t\rangle$ is a 2-sphere, where $\langle t\rangle$ denotes the cyclic group generated by the deck transformation $t$. We also denote by $C_{h}(t)$ the centralizer of $t$ in the diffeomorphism group Diff $+\Sigma_{h}$. We call the path-connected component $\pi_{0} C_{h}(t)$ the symmetric mapping class group of the covering $p$, which is defined by Birman and Hilden [1973].

In this section, we introduce 2-cocycles on the symmetric mapping class group $\pi_{0} C_{h}(t)$, derived from the nonadditivity formula for signatures. These are almost the same as the $\omega$-signatures defined in [Gambaudo and Ghys 2005].

Let us consider an oriented $\Sigma_{h}$-bundle $E_{\varphi, \psi}$ over $P$ whose structure group is contained in $C_{h}(t)$, and monodromies along $\alpha$ and $\beta$ are $\varphi$ and $\psi$ in $\pi_{0} C_{h}(t)$, respectively. Since coordinate transformations commute with the deck transformation $t$, we can define a fiberwise $\mathbb{Z} / d \mathbb{Z}$-action on $E_{\varphi, \psi}$. Since the structure group is in $C_{h}(t)$, not only $H_{1}\left(\Sigma_{h} ; \mathbb{C}\right)$ but also each eigenspace $V^{\eta^{j}}$ is a local system on $P$. We can extend the intersection form as a skew-hermitian form $H_{1}\left(\Sigma_{h} ; \mathbb{C}\right) \otimes H_{1}\left(\Sigma_{h} ; \mathbb{C}\right) \rightarrow \mathbb{C}$ defined by

$$
\left(x_{1}+x_{2} \boldsymbol{i}\right) \cdot\left(y_{1}+y_{2} \boldsymbol{i}\right)=x_{1} \cdot y_{1}+x_{2} \cdot y_{2}+\left(x_{1} \cdot y_{2}-x_{2} \cdot y_{1}\right) \boldsymbol{i} .
$$

For $v \in V^{\eta^{j}}$ and $w \in V^{\eta^{k}}(1 \leq j \leq d-1,1 \leq k \leq d-1)$,

$$
\begin{aligned}
& (t v) \cdot w=\left(\omega^{j} v\right) \cdot w=\omega^{-j}(v \cdot w), \\
& (t v) \cdot w=v \cdot\left(t^{-1} w\right)=v \cdot\left(\omega^{-k} w\right)=\omega^{-k}(v \cdot w) .
\end{aligned}
$$

Since $\omega^{-j}$ is not equal to $\omega^{-k}$, we have $v \cdot w=0$. Hence, $H_{1}\left(\Sigma_{h} ; \mathbb{C}\right)$ decomposes into an orthogonal sum of subspaces $\left\{V^{\omega^{j}}\right\}_{j=1}^{d-1}$. By restricting the intersection form on $H_{1}\left(\Sigma_{h} ; \mathbb{C}\right)$ to $V^{\eta^{j}}$, we can define a hermitian form on $H_{1}\left(P ; V^{\eta^{j}}\right)$. By Theorem 2.9, we have a 2-cocycle on $\pi_{0} C_{h}(t)$ as follows.

Lemma 3.1. Let $j$ be an integer such that $1 \leq j \leq m-1$. The map

$$
\tau_{m, d, j}: \pi_{0} C_{h}(t) \times \pi_{0} C_{h}(t) \rightarrow \mathbb{Z}
$$

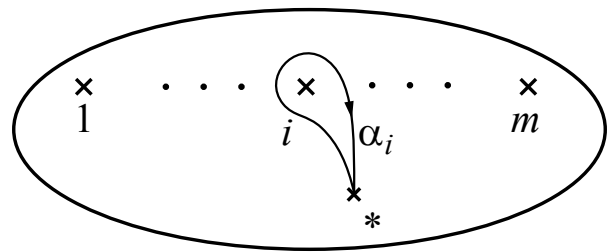

Figure 4. A loop $\alpha_{i}$. 
defined by

$$
\tau_{m, d, j}(\varphi, \psi)=\operatorname{Sign}\left(H_{1}\left(P ; V^{\eta^{j}}\right)\right)
$$

is a 2-cocycle, where $V^{\eta^{j}}$ is the local system on $P$ induced from the oriented $\Sigma_{h}$-bundle $E_{\varphi, \psi} \rightarrow P$.

Proof. The proof is the same as for [Meyer 1972, p. 43, Equation (0)]. Applying additivity of signatures to two oriented $\Sigma_{h}$-bundles on $P$, we can see that $\tau_{m, d, j}$ satisfies

$$
\tau_{m, d, j}\left(\varphi_{1}, \varphi_{2}\right)+\tau_{m, d, j}\left(\varphi_{1} \varphi_{2}, \varphi_{3}\right)=\tau_{m, d, j}\left(\varphi_{1}, \varphi_{2} \varphi_{3}\right)+\tau_{m, d, j}\left(\varphi_{2}, \varphi_{3}\right),
$$

for $\varphi_{1}, \varphi_{2}, \varphi_{3} \in \pi_{0} C_{h}(t)$.

Since the deck transformation $t$ acts on $H^{1}\left(P, \partial P ; V^{\eta^{j}}\right)$ by multiplication of $\eta^{j}$, we can calculate $\mathbb{Z} / d \mathbb{Z}$-signature as

$$
\operatorname{Sign}\left(H_{1}\left(P ; V^{\eta^{j}}\right), t^{k}\right)=\eta^{k j} \operatorname{Sign}\left(H_{1}\left(P ; V^{\eta^{j}}\right)\right)=\eta^{k j} \tau_{m, d, j}(\varphi, \psi),
$$

for $0 \leq k \leq m-1$. Moreover Meyer [1972, Satz I.2.2] $\operatorname{proved} \operatorname{Sign}\left(E_{\varphi, \psi}, t^{k}\right)=$ $\operatorname{Sign}\left(H_{1}\left(P ; H^{1}\left(\Sigma_{h} ; \mathbb{C}\right)\right), t^{k}\right)$. Hence we have:

Lemma 3.2. For $0 \leq k \leq m-1$,

$$
\operatorname{Sign}\left(E_{\varphi, \psi}, t^{k}\right)=\sum_{j=1}^{d-1} \eta^{k j} \tau_{m, d, j}(\varphi, \psi) .
$$

The symmetric mapping class groups. A diffeomorphism $f: \Sigma_{h} \rightarrow \Sigma_{h}$ in $C_{h}(t)$ induces a diffeomorphism $\bar{f}: S^{2} \rightarrow S^{2}$ which satisfies the commutative diagram

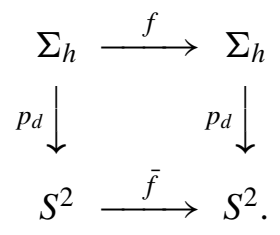

Moreover since $\bar{f}$ satisfies $p_{d}^{-1}(q)=p_{d}^{-1}(\bar{f}(q))$ for any $q \in S^{2}$, we have

$$
\bar{f} \in \operatorname{Diff}_{+}\left(S^{2},\left\{q_{i}\right\}_{i=1}^{m}\right) .
$$

Therefore we have a natural homomorphism $\mathscr{P}: \pi_{0} C_{h}(t) \rightarrow M_{0}^{m}$ which maps $[f]$ to $[\bar{f}]$. By a method similar to [Birman and Hilden 1971, Theorem 1] (see also [Birman and Hilden 1973, Section 5]), we have:

Lemma 3.3. Let $m \geq 4$. The sequence

$$
1 \longrightarrow \mathbb{Z} / d \mathbb{Z} \longrightarrow \pi_{0} C_{h}(t) \stackrel{\mathscr{P}}{\longrightarrow} M_{0}^{m} \longrightarrow 1
$$

is exact. 


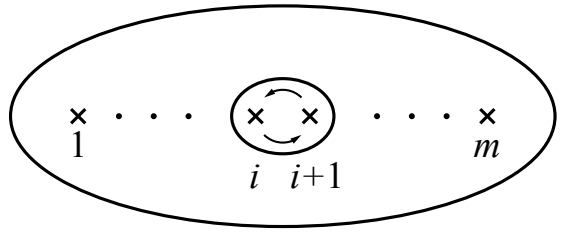

Figure 5. The diffeomorphism $s_{i}$.

Let $s_{i}: S^{2} \rightarrow S^{2}$ be a half twist of the disk which exchanges the points $q_{i}$ and $q_{i+1}$ as in Figure 5.

We denote by $\sigma_{i} \in \mathcal{M}_{0}^{m}$ the mapping class represented by $s_{i}$. By lifting $s_{i}$, we have a unique diffeomorphism $\tilde{s}_{i}: \Sigma_{h} \rightarrow \Sigma_{h}$ which satisfies supp $\tilde{s}_{i}=p_{d}^{-1}\left(\operatorname{supp} s_{i}\right)$. Let us denote the mapping class of $\tilde{s}_{i}$ by $\tilde{\sigma}_{i} \in \pi_{0} C_{h}(t)$. Note that when $d=2, \tilde{\sigma}_{i}$ is the Dehn twist along a nonseparating simple closed curve.

Lemma 3.4. The set $\left\{\tilde{\sigma}_{i}\right\}_{i=1}^{m-1} \subset \pi_{0} C_{h}(t)$ generates the group $\pi_{0} C_{h}(t)$.

Proof. Since $\left\{\sigma_{i}\right\}_{i=1}^{m-1}$ generates the group $\mu_{0}^{m}$, it suffices to represent $[t] \in \pi_{0} C_{h}(t)$ as a product of $\left\{\sigma_{i}\right\}_{i=1}^{m-1}$. Let $C_{h}^{(*)}(t)$ denote the subgroup of $C_{h}(t)$ defined by $C_{h}^{(*)}(t)=\left\{f \in C_{h}(t) \mid f\left(p_{d}^{-1}(*)\right)=p_{d}^{-1}(*)\right\}$. In this proof, by abuse of terminology, we use the term "Dehn twist" both for a diffeomorphism and for a mapping class. The diffeomorphism $s_{1} \cdots s_{m-2} s_{m-1}^{2} s_{m-2} \cdots s_{1}$ in $\operatorname{Diff}_{+}\left(S^{2},\left\{q_{i}\right\}_{i=1}^{m}\right)$ is isotopic to the product of Dehn twists $t_{c}^{-1} t_{c^{\prime}}$ in Figure 6, and it is also isotopic to the Dehn twist $t_{d}^{-1}$.

Therefore the lift $\tilde{s}_{1} \cdots \tilde{s}_{m-2} \tilde{s}_{m-1}^{2} \tilde{s}_{m-2} \cdots \tilde{s}_{1}$ is isotopic to some lift $\tilde{f}_{1}: \Sigma_{h} \rightarrow \Sigma_{h}$ of $t_{d}^{-1}$. Since we can choose the isotopy in $\operatorname{Diff}_{+}\left(S^{2},\left\{q_{i}\right\}_{i=1}^{m}\right)$ so that it does not move $*$, the lift $\tilde{f}_{1}$ fixes $p^{-1}(*)$ pointwise. Let $D$ be the closed disk which is bounded by $d$ and contains $*$, and let $\tilde{f}_{2}$ denote the lift of $t_{d}$ which satisfies supp $\tilde{f}_{2} \subset p^{-1}(D)$. Since $f_{1} f_{2}$ is a lift of the identity map of $S^{2}$, and the action of $\tilde{f}_{2}$ on $p^{-1}(*)$ coincides with that of $t$, we have $\tilde{f}_{1} \tilde{f}_{2}=t \in \operatorname{Diff}_{+} \Sigma_{h}$. Since $t_{d}$ is isotopic to the identity map in $\operatorname{Diff}_{+} \Sigma_{h}$, we have $\left[\tilde{f}_{2}\right]=1 \in \pi_{0} C_{h}(t)$. Thus we obtain

$$
\tilde{\sigma}_{1} \cdots \tilde{\sigma}_{m-2} \tilde{\sigma}_{m-1}^{2} \tilde{\sigma}_{m-2} \cdots \tilde{\sigma}_{1}=\left[\tilde{f}_{1}\right]=\left[\tilde{f}_{1} \tilde{f}_{2}\right]=[t] \in \pi_{0} C_{h}(t) .
$$
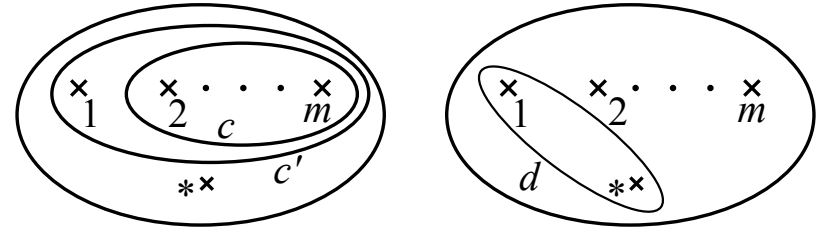

Figure 6. The curves $c, c^{\prime}, d$. 
The cobounding function of the cocycles $\tau_{m, d, j}$. Recall that, for an integer $d$ with $d \mid m$, we have a covering space $p_{d}: \Sigma_{h} \rightarrow S^{2}$. Let $g=(m-1)(m-2) / 2$. If we consider the case when $d=m, p_{d}$ is the $m$-cyclic covering on $S^{2}$ whose genus of the covering surface is $g$. Thus we identify it with the surface $\Sigma_{g}$, and denote the covering by $p: \Sigma_{g} \rightarrow S^{2}$.

Since the quotient space $\Sigma_{g} /\left\langle t^{d}\right\rangle$ is also a $d$-cyclic covering of $S^{2}$ with $m$ branched points, we can identify $\Sigma_{h} \cong \Sigma_{g} /\left\langle t^{d}\right\rangle$. Since a diffeomorphism $f \in C_{g}(t)$ induces a diffeomorphism $\bar{f}$ on $\Sigma_{g} /\left\langle t^{d}\right\rangle$ which commutes with $t$, we have a natural homomorphism $\mathscr{P}: \pi_{0} C_{g}(t) \rightarrow \pi_{0} C_{h}(t)$ which maps $[f]$ to $[\bar{f}]$. Since $H^{*}\left(\pi_{0} C_{h}(t) ; \mathbb{Q}\right) \cong H^{*}\left(\mathcal{M}_{0}^{m} ; \mathbb{Q}\right)$, and $H^{*}\left(\mathcal{M}_{0}^{m} ; \mathbb{Q}\right)$ is trivial (see [Cohen 1987] Corollary 2.2), there exists a unique cobounding function of $\tau_{m, d, j}$. Denote it by $\phi_{m, d, j}: \pi_{0} C_{h}(t) \rightarrow \mathbb{Q}$. Since $\tau_{m, d, j}$ is bounded, the cobounding function $\phi_{m, d, j}$ is a quasimorphism.

Remark 3.5. Gambaudo and Ghys [2005] already constructed almost the same quasimorphisms on the mapping class groups of pointed disks, called $\omega$-signatures. They calculated the value of their quasimorphisms for an element similar to

$$
\tilde{\sigma}_{1} \tilde{\sigma}_{2} \cdots \tilde{\sigma}_{r-1} \in \pi_{0} C_{h}(t)
$$

in [Gambaudo and Ghys 2005, Proposition 5.2].

Remark 3.6. This construction is also similar to higher-order signature cocycles in Cochran, Harvey and Horn's paper [Cochran et al. 2012]. They considered von Neumann signatures of surface bundles whose fibers are nonfinite regular coverings on a surface with boundary.

Let us recall a natural homomorphism $\pi_{0} C_{h}(t) \rightarrow \mu_{h}$ defined by forgetting symmetries of mapping classes. It maps a mapping class $[f] \in \pi_{0} C_{h}(t)$ to $[f] \in \mathcal{M}_{h}$, and is injective as shown in Birman and Hilden [1973, Theorem 1]. In particular, if we consider the case when $m$ is even and the double covering $p_{2}: \Sigma_{h} \rightarrow S^{2}$, this homomorphism induces isomorphism between $\pi_{0} C_{h}(t)$ and $\mathscr{H}_{h}$. In this case, the eigenspace $V^{-1}$ coincides with $H_{1}\left(\Sigma_{h} ; \mathbb{C}\right)$. Thus we have:

Remark 3.7. When $m$ is even, $\phi_{m, 2,1}: \pi_{0} C_{h}(t) \rightarrow \mathbb{Q}$ is equal to the Meyer function $\phi_{h}: \mathscr{H}_{h} \rightarrow \mathbb{Q}$ on the hyperelliptic mapping class group, under the natural isomorphism $\pi_{0} C_{h}(t) \cong \mathscr{H}_{h}$.

Lemma 3.8. For $1 \leq j \leq d-1$ and $\varphi \in \pi_{0} C_{g}(t)$,

$$
\phi_{m, m, m j / d}(\varphi)=\phi_{m, d, j}(\mathscr{P}(\varphi)) .
$$

Proof. Since $H_{1}\left(\pi_{0} C_{g}(t) ; \mathbb{Q}\right)$ is trivial, it suffices to show that

$$
\tau_{m, m, m j / d}(\varphi, \psi)=\tau_{m, d, j}(\mathscr{P}(\varphi), \mathscr{P}(\psi)) \quad \text { for } \varphi, \psi \in \pi_{0} C_{g}(t) .
$$


If $f: E \rightarrow P$ is an oriented $\Sigma_{g}$-bundle with structure group $C_{g}(t)$, the induced map $\bar{f}: E /\left\langle t^{d}\right\rangle \rightarrow P$ is an oriented $\Sigma_{h}$-bundle with structure group $C_{h}(t)$. If we denote the monodromies of $f$ along $\alpha$ and $\beta$ by $\varphi$ and $\psi$, the ones of $\bar{f}$ are $\mathscr{P}(\varphi)$ and $\mathscr{P}(\psi)$.

Let $\omega$ be the $m$-th root of unity $\exp (2 \pi \boldsymbol{i} / m)$, and let $q_{d}: \Sigma_{g} \rightarrow \Sigma_{g} /\left\langle t^{d}\right\rangle$ denote the projection. To distinguish eigenspaces of $H_{1}\left(\Sigma_{g} ; \mathbb{C}\right)$ and $H_{1}\left(\Sigma_{h} ; \mathbb{C}\right)$ of the action by $t$, we denote them by $\left(V_{g}\right)^{z}$ and $\left(V_{h}\right)^{z}$ instead of $V^{z}$, respectively. The projection $q_{d}$ induces the isomorphism $H_{1}\left(\Sigma_{g} ; \mathbb{C}\right)^{\left\langle t^{d}\right\rangle} \cong H_{1}\left(\Sigma_{h} ; \mathbb{C}\right)$. Moreover we have $\left(V_{g}\right)^{\omega^{m j / d}} \cong\left(V_{h}\right)^{\eta^{j}}$. Hence it also induces a natural isomorphism between $H_{1}\left(P ;\left(V_{g}\right)^{\omega^{m j / d}}\right)$ and $H_{1}\left(P ;\left(V_{h}\right)^{\eta^{j}}\right)$, where $\left(V_{g}\right)^{\omega^{m j / d}}$ and $\left(V_{h}\right)^{\eta^{j}}$ are local systems coming from $f$ and $\bar{f}$.

Let $\tilde{a}, \tilde{b}$ be loops in $\Sigma_{g}-\left\{q_{i}\right\}_{i=1}^{m}$. We may assume that $q_{d}(\tilde{a}) \cup q_{d}(\tilde{b})$ has no triple point. Then the intersection number $\left[q_{d}(\tilde{a})\right] \cdot\left[q_{d}(\tilde{b})\right]$ in $\Sigma_{h}$ coincides with $\left[q_{d}^{-1}\left(q_{d}(\tilde{a})\right)\right] \cdot[\tilde{b}]$ in $\Sigma_{g}$. Hence we have

$$
\begin{aligned}
\sum_{i=0}^{m / d-1}\left[\left(t^{d i}\right)_{*} \tilde{a}\right] \cdot \sum_{j=0}^{m / d-1}\left[\left(t^{d j}\right)_{*} \tilde{b}\right] & =\sum_{i=0}^{m / d-1} \sum_{j=0}^{m / d-1}\left[\left(t^{d i-d j}\right)_{*} \tilde{a}\right] \cdot[\tilde{b}] \\
& =\frac{m}{d}\left[q_{d}^{-1}\left(q_{d}(\tilde{a})\right)\right] \cdot[\tilde{b}]=\frac{m}{d}\left[q_{d}(\tilde{a})\right] \cdot\left[q_{d}(\tilde{b})\right]
\end{aligned}
$$

Therefore the isomorphism $H_{1}\left(\Sigma_{g} ; \mathbb{C}\right)^{\left\langle t^{d}\right\rangle} \cong H_{1}\left(\Sigma_{h} ; \mathbb{C}\right)$ induced by the quotient map $q_{d}: \Sigma_{g} \rightarrow \Sigma_{h}$ preserves the intersection form up to constant multiple. Thus it also preserves the intersection forms on $H_{1}\left(P ;\left(V_{g}\right)^{\omega^{m j} / d}\right)$ and $H_{1}\left(P ;\left(V_{h}\right)^{\eta^{j}}\right)$, and we obtain

$$
\begin{aligned}
\tau_{m, m, m j / d}(\varphi, \psi) & =\operatorname{Sign}\left(H_{1}\left(P ;\left(V_{g}\right)^{\omega^{m j / d}}\right)\right) \\
& =\operatorname{Sign}\left(H_{1}\left(P ;\left(V_{h}\right)^{\eta^{j}}\right)\right) \\
& =\tau_{m, d, j}(\mathscr{P}(\varphi), \mathscr{P}(\psi)) .
\end{aligned}
$$

By Lemma 3.8, it suffices to consider the case when $d=m$. We shorten $\tau_{m, m, j}$ and $\phi_{m, m, j}$ to $\tau_{m, j}$ and $\phi_{m, j}$.

Lemma 3.9.

$$
\phi_{m, j}(\varphi)=\phi_{m, m-j}(\varphi) .
$$

Proof. By taking complex conjugates, we have an isomorphism $i: V^{\omega^{j}} \cong V^{\omega^{m-j}}$. Moreover it induces the isomorphism $i_{*}: H_{1}\left(P ; V^{\omega^{j}}\right) \cong H_{1}\left(P ; V^{\omega^{m-j}}\right)$.

Let us denote the hermitian form on $H_{1}\left(P ; V^{\omega^{j}}\right)$ by $\langle,\rangle_{j}$. By the definition

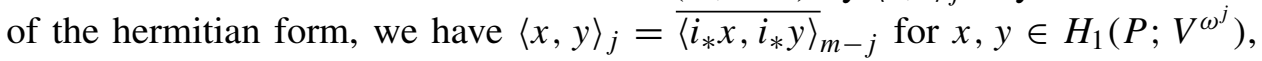
where $\bar{z}$ is a complex conjugate of $z \in \mathbb{C}$. Thus the signatures of the hermitian forms $\langle,\rangle_{j}$ and $\langle,\rangle_{m-j}$ coincide, and the cobounding functions of $\tau_{m, j}$ and $\tau_{m, m-j}$ also coincide. 


\section{Defects of homogeneous quasimorphisms}

In this section, we will prove Theorems 1.3 and 1.6. On page 336, we give an inequality between the defects of a quasimorphism and its homogenization when the quasimorphism is antisymmetric and a class function (Lemma 4.1) and prove Theorem 1.3(i). On page 337, we prove Theorem 1.3(ii) by giving a lower bound on the defect of $\phi_{m, m / 2}: \pi_{0} C_{g}(t) \rightarrow \mathbb{R}$, which is the cobounding function of the 2-cocycle $\tau_{m, m / 2}$. On page 337, we prove Theorem 1.6.

Proof of Theorem 1.3(i). Endo [2000, Proposition 3.1] showed that the Meyer function $\phi_{g}: \mathscr{H}_{g} \rightarrow \mathbb{Q}$ satisfies the conditions in Lemma 4.1. The quasimorphisms $\bar{\phi}_{m, j}$ also satisfy these conditions.

Turaev [1985] defined another 2-cocycle on the symplectic group. Endo and Nagami [2005, Proposition A.3] showed that Turaev's cocycle coincides with the Meyer cocycle up to sign. Since Turaev's cocycle is defined by the signature on a vector space of rank less than or equal to $m-2$. A similar argument shows $D\left(\phi_{m, j}\right) \leq m-2$. Thus Theorem 1.3(i) follows from Lemma 4.1 below.

Lemma 4.1. Let $G$ be a group, and $\phi: G \rightarrow \mathbb{R}$ a quasimorphism satisfying

$$
\phi\left(x y x^{-1}\right)=\phi(y), \quad \phi\left(x^{-1}\right)=-\phi(x) .
$$

Then we have

$$
D(\bar{\phi}) \leq D(\phi),
$$

where $\bar{\phi}$ is the homogenization of $\phi$.

Proof of Lemma 4.1. Without loss of generality, we may assume that the quasimorphism $\phi: G \rightarrow \mathbb{R}$ is antisymmetric:

$$
\phi\left(x^{-1}\right)=-\phi(x) .
$$

Otherwise pass to the antisymmetrization $\phi^{\prime}: G \rightarrow \mathbb{R}$ defined by

which satisfies

$$
\phi^{\prime}(x)=\frac{\phi(x)-\phi\left(x^{-1}\right)}{2},
$$

$$
D\left(\phi^{\prime}\right) \leq D(\phi) \text {, and } \bar{\phi}^{\prime}=\bar{\phi} .
$$

For any $x, y \in G$, we have

$$
\begin{aligned}
\phi([x, y]) & =|\phi([x, y])-\phi(y)+\phi(y)| \\
& =\left|\phi\left(x y x^{-1} y^{-1}\right)-\phi\left(x y x^{-1}\right)-\phi\left(y^{-1}\right)\right| \leq D(\phi) .
\end{aligned}
$$

Thus for any $g \in[G, G]$,

$$
|\phi(g)| \leq(2 \operatorname{cl}(g)-1) D(\phi) .
$$


As observed by Bavard [1991, Lemma 3.6],

$$
\operatorname{cl}\left(x^{n} y^{n}(x y)^{-n}\right) \leq \frac{n}{2},
$$

for every $n \geq 0$. Therefore we have $\left|\phi\left(x^{n} y^{n}(x y)^{-n}\right)\right| \leq(n-1) D(\phi)$. Hence

$$
\begin{aligned}
|\delta \bar{\phi}(x, y)| & =\lim _{n \rightarrow \infty}\left|\frac{\phi\left(x^{n}\right)+\phi\left(y^{n}\right)-\phi\left(x^{n} y^{n}\right)}{n}\right| \\
& =\lim _{n \rightarrow \infty}\left|\frac{\phi\left(x^{n} y^{n}(x y)^{-n}\right)}{n}\right| \leq D(\phi) .
\end{aligned}
$$

Proof of Theorem 1.3(ii). Let $m$ be an even number greater than or equal to 4. By Remark 3.7, we consider the Meyer function $\phi_{g}$ on the hyperelliptic mapping class group $\mathscr{H}_{g}$ instead of $\phi_{m, m / 2}$.

Lemma 4.2 [Barge and Ghys 1992, Proposition 3.5]. For any $A \in \operatorname{Sp}(2 g ; \mathbb{Z})$,

$$
\operatorname{Sign}\left(\langle,\rangle_{A^{k}, A}\right)=\operatorname{Sign}\left(-J \sum_{i=1}^{k}\left(A^{i}-A^{-i}\right)\right) .
$$

Let $c_{i}, d_{i}^{+}$, and $d_{i}^{-}$denote the simple closed curves in Figure 7. For simplicity, we also denote by $c_{i}, d_{i}^{+}$, and $d_{i}^{-}$the Dehn twists along these curves.

To prove Theorem 1.3(ii), it suffices to show the following.

Lemma 4.3. $\delta \bar{\phi}_{g}\left(c_{2}^{2} c_{4}^{2} \cdots c_{2 g}^{2}, d_{1}^{+} d_{1}^{-} d_{2}^{+} d_{2}^{-} \cdots d_{g}^{+} d_{g}^{-}\right)=-2 g$.

Proof of Lemma 4.3. Since the pairs $\left(c_{i}, c_{j}\right),\left(d_{i}^{+} d_{i}^{-}, d_{j}^{+} d_{j}^{-}\right)$, and $\left(c_{i}, d_{j}^{+} d_{j}^{-}\right)$ mutually commute when $i \neq j$, the expression in the lemma equals

$$
\begin{aligned}
\bar{\phi}_{g}\left(c_{2}^{2} c_{4}^{2} \cdots c_{2 g}^{2}\right)+\bar{\phi}_{g}\left(d_{1}^{+} d_{1}^{-} d_{2}^{+} d_{2}^{-} \cdots\right. & \left.d_{g}^{+} d_{g}^{-}\right)-\bar{\phi}_{g}\left(c_{2}^{2} d_{1}^{+} d_{1}^{-} c_{4}^{2} d_{2}^{+} d_{2}^{-} \cdots c_{2 g}^{2} d_{g}^{+} d_{g}^{-}\right) \\
& =\sum_{i=1}^{g}\left(\bar{\phi}_{g}\left(c_{2 i}^{2}\right)+\bar{\phi}_{g}\left(d_{i}^{+} d_{i}^{-}\right)-\bar{\phi}_{g}\left(c_{2 i}^{2} d_{i}^{+} d_{i}^{-}\right)\right) .
\end{aligned}
$$

Hence it suffices to prove $\bar{\phi}_{g}\left(c_{2 i}^{2}\right)+\bar{\phi}_{g}\left(d_{i}^{+} d_{i}^{-}\right)-\bar{\phi}_{g}\left(c_{2 i}^{2} d_{i}^{+} d_{i}^{-}\right)=-2$ for $1 \leq i \leq g$.

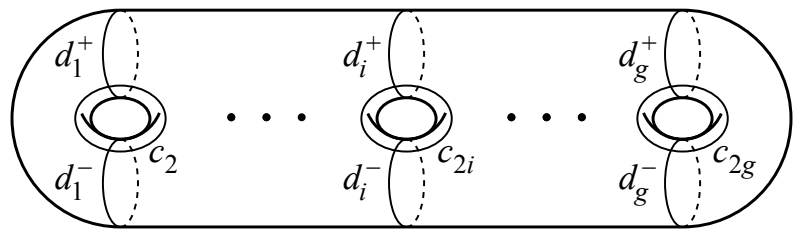

Figure 7. Curves in $\Sigma_{g}$. 
Since $\rho\left(d_{i}^{+}\right)=\rho\left(d_{i}^{-}\right)$, we have

$$
\begin{aligned}
\bar{\phi}_{g}\left(c_{2 i}^{2}\right)+\bar{\phi}_{g}\left(d_{i}^{+} d_{i}^{-}\right)-\bar{\phi}_{g}\left(c_{2 i}^{2} d_{i}^{+} d_{i}^{-}\right) & \\
=-\lim _{n \rightarrow \infty} \frac{1}{n}\left\{\phi_{g}\left(\left(c_{2 i}^{2} d_{i}^{+} d_{i}^{-}\right)^{n}\right)-\phi_{g}\left(\left(c_{2 i}^{2}\right)^{n}\right)-\phi_{g}\left(\left(d_{i}^{+} d_{i}^{-}\right)^{n}\right)\right\} & \\
=\lim _{n \rightarrow \infty} \frac{1}{n} \sum_{k=1}^{n-1}\left\{\tau_{g}\left(\left(c_{2 i}^{2} d_{i}^{+} d_{i}^{-}\right)^{k}, c_{2 i}^{2} d_{i}^{+} d_{i}^{-}\right)-\tau_{g}\left(c_{2 i}^{2 i}, c_{2 i}^{2}\right)-\tau_{g}\left(\left(d_{i}^{+} d_{i}^{-}\right)^{i}, d_{i}^{+} d_{i}^{-}\right)\right\} & +\tau_{g}\left(c_{2 i}^{2}, d_{i}^{+} d_{i}^{-}\right)
\end{aligned}
$$

There exists a mapping class $\psi_{i}$ such that $\psi_{i} c_{2 i} \psi_{i}^{-1}=c_{2}$ and $\psi_{i} d_{i}^{+} \psi_{i}^{-1}=d_{i}^{+}$ for $i=2, \ldots, g$. Since the Meyer cocycle satisfies the property

$$
\tau_{g}\left(x y x^{-1}, x z x^{-1}\right)=\tau_{g}(y, z)
$$

for $x, y, z \in M_{g}$, we have

$$
\begin{aligned}
& \lim _{n \rightarrow \infty} \sum_{k=1}^{n-1} \frac{1}{n}\left\{\tau_{g}\left(\left(c_{2 i}^{2}\left(d_{i}^{+}\right)^{2}\right)^{k}, c_{2 i}^{2}\left(d_{i}^{+}\right)^{2}\right)-\tau_{g}\left(c_{2 i}^{2 i}, c_{2 i}^{2}\right)-\tau_{g}\left(\left(d_{i}^{+}\right)^{2 i},\left(d_{i}^{+}\right)^{2}\right)\right\}+\tau_{g}\left(c_{2 i}^{2},\left(d_{i}^{+}\right)^{2}\right) \\
&=\lim _{n \rightarrow \infty} \sum_{k=1}^{n-1} \frac{1}{n}\left\{\tau_{g}\left(\left(c_{2}^{2}\left(d_{1}^{+}\right)^{2}\right)^{k}, c_{2}^{2}\left(d_{1}^{+}\right)^{2}\right)-\tau_{g}\left(c_{2}^{2 i}, c_{2}^{2}\right)-\tau_{g}\left(\left(d_{1}^{+}\right)^{2 i},\left(d_{1}^{+}\right)^{2}\right)\right\} \\
&+ \\
&+\tau_{g}\left(c_{2}^{2},\left(d_{1}^{+}\right)^{2}\right) .
\end{aligned}
$$

Let us consider the case when $g=1$. Since $\rho\left(c_{2}^{2}\right)=\left(\begin{array}{ll}1 & 2 \\ 0 & 1\end{array}\right), \rho\left(\left(d_{1}^{+}\right)^{2}\right)=\left(\begin{array}{rr}1 & 0 \\ -2 & 1\end{array}\right)$ and $\rho\left(c_{2}^{2}\left(d_{1}^{+}\right)^{2}\right)=\left(\begin{array}{ll}-3 & 2 \\ -2 & 1\end{array}\right)$, we have

$$
\begin{aligned}
-J \sum_{k=1}^{n}\left(\rho\left(c_{2}^{2 k}\right)-\rho\left(c_{2}^{-2 k}\right)\right) & =\left(\begin{array}{ll}
0 & 0 \\
0 & 2 n(n+1)
\end{array}\right), \\
-J \sum_{k=1}^{n}\left(\rho\left(d_{1}^{+}\right)^{2 k}-\rho\left(d_{1}^{+}\right)^{-2 k}\right) & =\left(\begin{array}{cc}
2 n(n+1) & 0 \\
0 & 0
\end{array}\right), \\
-J \sum_{k=1}^{n}\left(\rho\left(\left(c_{2}^{2}\left(d_{1}^{+}\right)^{2}\right)^{k}\right)-\rho\left(\left(c_{2}^{2}\left(d_{1}^{+}\right)^{2}\right)^{-k}\right)\right) & =\sum_{k=1}^{n} 4 k(-1)^{k}\left(\begin{array}{rr}
-1 & 1 \\
1 & -1
\end{array}\right) \\
& =\left\{(-1)^{n}(2 n+1)-1\right\}\left(\begin{array}{rr}
-1 & 1 \\
1 & -1
\end{array}\right) .
\end{aligned}
$$

By Lemma 4.2 we obtain 
(1)

$$
\begin{aligned}
& \lim _{n \rightarrow \infty} \sum_{k=1}^{n-1} \frac{\tau_{g}\left(c_{2}^{2 k}, c_{2}^{2}\right)}{n}=\lim _{n \rightarrow \infty} \sum_{k=1}^{n-1} \frac{\tau_{g}\left(\left(d_{1}^{+}\right)^{2 k},\left(d_{1}^{+}\right)^{2}\right)}{n}=1, \\
& \lim _{n \rightarrow \infty} \sum_{k=1}^{n-1} \frac{\tau_{g}\left(\left(c_{2}^{2}\left(d_{1}^{+}\right)^{2}\right)^{k}, c_{2}^{2}\left(d_{1}^{+}\right)^{2}\right)}{n}=0 .
\end{aligned}
$$

When $g \geq 2$, the same calculation also shows (1). It is an easy calculation to show that

$$
\tau_{g}\left(c_{2}^{2}, d_{1}^{+} d_{1}^{-}\right)=0
$$

Therefore we obtain

$$
\bar{\phi}_{g}\left(c_{2 i}^{2}\right)+\bar{\phi}_{g}\left(d_{i}^{+} d_{i}^{-}\right)-\bar{\phi}_{g}\left(c_{2 i}^{2} d_{i}^{+} d_{i}^{-}\right)=-2 .
$$

In the same way as for $(1)$, we have $\tau_{g}\left(s_{0}^{i}, s_{0}\right)=1$. Hence we obtain

$$
\bar{\phi}_{g}\left(s_{0}\right)=-\lim _{n \rightarrow \infty} \frac{\sum_{i=1}^{n-1} \tau_{g}\left(s_{0}^{i}, s_{0}\right)}{n}+\phi_{g}\left(s_{0}\right)=-1+\phi_{g}\left(s_{0}\right)
$$

and

$$
\bar{\phi}_{g}\left(s_{h}\right)=\phi_{g}\left(s_{0}\right) \text {. }
$$

By [Endo 2000, Lemmas 3.3 and 3.5], we have

$$
\bar{\phi}_{g}\left(t_{s_{0}}\right)=-\frac{g}{2 g+1}, \quad \text { and } \quad \bar{\phi}_{g}\left(t_{s_{h}}\right)=-\frac{4 h(g-h)}{2 g+1} .
$$

Remark 4.4. By Theorems 1.3 and 2.4, $\bar{\phi}_{g}$ gives the lower bounds for $\operatorname{scl}_{\mathscr{H}_{g}}\left(t_{s_{h}}\right)$ $(j=0, \ldots, g-1)$ corresponding to ones given in [Monden 2012].

Remark 4.5. By Theorems 1.7 and 2.4 and Remark 4.4, we have $\operatorname{scl}_{\mathcal{M}_{1}}\left(t_{c}\right)=\frac{1}{12}$. Let $\rho: M_{1} \cong \operatorname{SL}(2, \mathbb{Z}) \rightarrow \operatorname{PSL}(2, \mathbb{Z})$ be the natural quotient map. It is easily seen that for all $x \in \mathcal{M}_{1}, \operatorname{scl}_{\mathcal{M}_{1}}(x)=\operatorname{scl}_{\operatorname{PSL}(2, \mathbb{Z})}(\rho(x))$. Louwsma [2011] determined $\operatorname{scl}_{\operatorname{PSL}(2, \mathbb{Z})}(y)=\frac{1}{12}$ for $y=\rho\left(t_{c}\right)$.

Proof of Theorem 1.6. If $x \in \mathscr{H}_{g}$ satisfies $\left|\bar{\phi}_{g}(x)\right|=D\left(\bar{\phi}_{g}\right)$ and $|\phi(x)| \leq D(\phi)$ for any homogeneous quasimorphism $\phi: \mathscr{H}_{g} \rightarrow \mathbb{R}$, we obtain $\operatorname{scl}(x)=\frac{1}{2}$ by Bavard's duality theorem (Theorem 2.4). We will show that

$$
x=c^{2 g+8}\left(d_{2}^{+} d_{2}^{-} \cdots d_{g-1}^{+} d_{g-1}^{-}\right)^{2}\left(s_{1} \cdots s_{g-1}\right)^{-1}
$$

satisfies this property.

Firstly we will prove

$$
\sum_{j=1}^{g-1} \phi\left(s_{j}\right)=\sum_{i=1}^{g}\left(\phi\left(c_{2 i}^{2} d_{i}^{-} d_{i}^{+}\right)-\phi\left(d_{i}^{-} d_{i}^{+}\right)\right)
$$


for any homogeneous quasimorphism $\phi: \mathscr{H}_{g} \rightarrow \mathbb{R}$. By Lemma 2.8, we have

$$
\begin{gathered}
\left(d_{1}^{+} c_{2} d_{1}^{-}\right)^{4}=s_{1}, \quad\left(d_{g}^{+} c_{2 g} d_{g}^{-}\right)^{4}=s_{g-1}, \\
\left(d_{i}^{+} c_{2 i} d_{i}^{-}\right)^{4}=s_{i-1} s_{i} \quad(i=2, \ldots, g-1) .
\end{gathered}
$$

Since $c_{2 i}$ commutes with $s_{j},\left(c_{2} d_{1}^{-} d_{1}^{+} c_{2} d_{1}^{-} d_{1}^{+}\right)^{2}=s_{1},\left(c_{2 i} d_{i}^{-} d_{i}^{+} c_{2 i} d_{i}^{-} d_{i}^{+}\right)^{2}=s_{i-1} s_{i}$, and $\left(c_{2 g} d_{g}^{-} d_{g}^{+} c_{2 g} d_{g}^{-} d_{g}^{+}\right)^{2}=s_{g-1}$. By Lemma 2.6, $c_{2 i} d_{i}^{-} d_{i}^{+} c_{2 i}$ commutes with $d_{i}^{-} d_{i}^{+}$for $i=1, \ldots, g$, as is easy to check. Therefore $\left(c_{2} d_{1}^{-} d_{1}^{+} c_{2}\right)^{2}=s_{1}\left(d_{1}^{-} d_{1}^{+}\right)^{-2}$, $\left(c_{2 i} d_{i}^{-} d_{i}^{+} c_{2 i}\right)^{2}=s_{i-1} s_{i}\left(d_{i}^{-} d_{i}^{+}\right)^{-2}$, and $\left(c_{2 g} d_{g}^{-} d_{g}^{+} c_{2 g}\right)^{2}=s_{g-1}\left(d_{g}^{-} d_{g}^{+}\right)^{-2}$. These equations give

$$
\begin{aligned}
2 \phi\left(c_{2}^{2} d_{1}^{-} d_{1}^{+}\right) & =\phi\left(s_{1}\right)-2 \phi\left(d_{1}^{-} d_{1}^{+}\right), \\
2 \phi\left(c_{2 i}^{2} d_{i}^{-} d_{i}^{+}\right) & =\phi\left(s_{i-1}\right)+\phi\left(s_{i}\right)-2 \phi\left(d_{i}^{-} d_{i}^{+}\right), \\
2 \phi\left(c_{2 g}^{2} d_{g}^{-} d_{g}^{+}\right) & =\phi\left(s_{g-1}\right)-2 \phi\left(d_{g}^{-} d_{g}^{+}\right) .
\end{aligned}
$$

Thus we obtain $\sum_{j=1}^{g-1} \phi\left(s_{j}\right)=\sum_{i=1}^{g}\left(\phi\left(c_{2 i}^{2} d_{i}^{-} d_{i}^{+}\right)-\phi\left(d_{i}^{-} d_{i}^{+}\right)\right)$.

Secondly we will prove $\bar{\phi}_{g}(x)=D\left(\bar{\phi}_{g}\right)$. The curves $c, s_{1}, \ldots, s_{g-1}, d_{2}^{+}, d_{2}^{-}, \ldots$, $d_{g-1}^{+}, d_{g-1}^{-}$are mutually disjoint, and $c_{i}$ is conjugate to $c$. Hence, by Lemma 2.3(i) and (ii), we have

$$
\begin{aligned}
\phi(x) & =(g+4) \phi\left(c^{2}\right)+2 \sum_{i=2}^{g-1} \phi\left(d_{i}^{+} d_{i}^{-}\right)-\sum_{j=1}^{g-1} \phi\left(s_{i}\right) \\
& =\sum_{i=1}^{g}\left(\phi\left(c_{2 i}^{2}\right)+\phi\left(d_{i}^{+} d_{i}^{-}\right)-\phi\left(c_{2 i}^{2} d_{i}^{-} d_{i}^{+}\right)\right) .
\end{aligned}
$$

In the proof of Lemma 4.3, we showed

$$
\sum_{i=1}^{g}\left(\bar{\phi}_{g}\left(c_{2 i}^{2}\right)+\bar{\phi}_{g}\left(d_{i}^{+} d_{i}^{-}\right)-\bar{\phi}_{g}\left(c_{2 i}^{2} d_{i}^{-} d_{i}^{+}\right)\right)=-2 g=-D\left(\bar{\phi}_{g}\right) .
$$

Thus we obtain $\left|\bar{\phi}_{g}(x)\right|=D\left(\bar{\phi}_{g}\right)$.

Lastly we prove $\phi(x) \leq D(\phi)$ for any homogeneous quasimorphism $\phi: \mathscr{H}_{g} \rightarrow \mathbb{R}$ :

$$
\begin{aligned}
D(\phi) & \geq\left|\delta\left(c_{2}^{2} \cdots c_{2 g}^{2}, d_{1}^{+} d_{1}^{-} \cdots d_{g}^{+} d_{g}^{-}\right)\right| \\
& =\left|\phi\left(c_{2}^{2} \cdots c_{2 g}^{2}\right)+\phi\left(d_{1}^{+} d_{1}^{-} \cdots d_{g}^{+} d_{g}^{-}\right)-\phi\left(c_{2}^{2} \cdots c_{2 g}^{2} d_{1}^{+} d_{1}^{-} \cdots d_{g}^{+} d_{g}^{-}\right)\right| \\
& =\left|\phi\left(c_{2}^{2} \cdots c_{2 g}^{2}\right)+\phi\left(d_{1}^{+} d_{1}^{-} \cdots d_{g}^{+} d_{g}^{-}\right)-\phi\left(\left(c_{2}^{2} d_{1}^{+} d_{1}^{-}\right) \cdots\left(c_{2 g}^{2} d_{g}^{+} d_{g}^{-}\right)\right)\right| \\
& =\left|\sum_{i=1}^{g}\left(\phi\left(c_{2 i}^{2}\right)+\phi\left(d_{i}^{+} d_{i}^{-}\right)-\phi\left(c_{2 i}^{2} d_{i}^{+} d_{i}^{-}\right)\right)\right|=|\phi(x)| .
\end{aligned}
$$




\section{Proof of Theorem 1.7}

Let $c_{1}, \ldots, c_{2 g+2}$ be nonseparating simple closed curves on $\Sigma_{g}$ as in Figure 2 and let $\phi$ be a homogeneous quasimorphism on $\mathscr{H}_{g}$. For simplicity of notation, we write $t_{i}$ instead of $t_{c_{i}}$ By $\iota=\iota^{-1}$, we have $t_{2 g+1}^{2} t_{2 g} \cdots t_{2} t_{1}^{2}=\left(t_{2 g} \cdots t_{2}\right)^{-1} \iota^{-1}$. Since each of the two boundary components of a regular neighborhood of $c_{2} \cup c_{3} \cup \cdots \cup c_{2 g}$ is $c_{2 g+2}$ by Lemma 2.8 , we have $\left(t_{2 g} \cdots t_{2}\right)^{2 g}=t_{2 g+2}^{2}$. Note that this relation holds in $\mathscr{H}_{g}$. Therefore, by Definition 2.2 and Lemma 2.3, we have

$$
\phi\left(t_{2 g+1}^{2} t_{2 g} \cdots t_{2} t_{1}^{2}\right)=-\phi\left(t_{2 g} \cdots t_{2}\right)+\phi\left(\iota^{-1}\right)=-\frac{1}{g} \phi\left(t_{2 g+2}\right) .
$$

Applying Lemma 2.3(i) and 2.6(i), we can move the factors with single and double underlines alternatively as follows.

$$
\begin{aligned}
& \phi\left(t_{2 g+1}^{2} t_{2 g} \cdots t_{3} t_{2} t_{1}^{2}\right)=\phi\left(t_{1}^{2} t_{2 g+1}^{2} t_{2 g} \cdots t_{3} t_{2}\right) \\
& =\phi\left(t_{2 g+1}^{2} t_{2 g} \cdots t_{3} \underline{t_{1}^{2}} \underline{\underline{t_{2}}}\right) \\
& =\phi\left(\underline{\underline{t}}_{2} t_{2 g+1}^{2} t_{2 g} \cdots t_{4} t_{3} t_{1}^{2}\right) \\
& =\phi\left(t_{2 g+1}^{2} t_{2 g} \cdots t_{4} \underline{t_{2}} \underline{t_{3}} t_{1}^{2}\right) \\
& =\phi\left(t_{2 g+1}^{2} t_{2 g} \cdots t_{6} t_{5} \underline{t_{3} t_{1}^{2}} \underline{\underline{t_{4} t_{2}}}\right) \\
& =\phi\left(t_{2 g+1}^{2} t_{2 g} \cdots t_{6} \underline{\underline{t_{4}} t_{2}} t_{5} t_{3} t_{1}^{2}\right) \\
& =\phi\left(t_{2 g+1}^{2} t_{2 g} \cdots t_{7} t_{5} t_{3} t_{1}^{2} \underline{t_{6} t_{4} t_{2}}\right) \\
& =\phi\left(t_{2 g+1}^{2} t_{2 g} \cdots t_{8} t_{6} t_{4} t_{2} t_{7} t_{5} t_{3} t_{1}^{2}\right) \\
& =\phi\left(t_{2 g+1}^{2} t_{2 g} \cdots t_{9} t_{7} t_{5} t_{3} t_{1}^{2} t_{8} t_{6} t_{4} t_{2}\right) \\
& \text { (by Lemma 2.3) } \\
& \text { (by Lemma 2.6) } \\
& \text { (by Lemma 2.3) } \\
& \text { (by Lemma 2.6) } \\
& \text { (by Lemma } 2.3 \text { and 2.6) } \\
& \text { (by Lemmas } 2.3 \text { and 2.6) } \\
& \text { (by Lemmas } 2.3 \text { and 2.6) } \\
& \text { (by Lemmas } 2.3 \text { and 2.6) } \\
& \text { (by Lemmas } 2.3 \text { and 2.6) } \\
& =\phi\left(\left(t_{2 g+1}^{2} t_{2 g-1} \cdots t_{5} t_{3} t_{1}^{2}\right)\left(t_{2 g} t_{2 g-4} \cdots t_{4} t_{2}\right)\right) \text {. }
\end{aligned}
$$

From Definition 2.2 and Equation (3),

$$
\begin{aligned}
D(\phi) & \geq\left|\phi\left(\left(t_{2 g+1}^{2} \cdots t_{3} t_{1}^{2}\right)\left(t_{2 g} \cdots t_{4} t_{2}\right)\right)-\phi\left(t_{2 g+1}^{2} \cdots t_{3} t_{1}^{2}\right)-\phi\left(t_{2 g} \cdots t_{4} t_{2}\right)\right| \\
& =\left|-\frac{1}{g} \phi\left(t_{2 g+2}\right)-\phi\left(t_{2 g+1}^{2} \cdots t_{3} t_{1}^{2}\right)-\phi\left(t_{2 g} \cdots t_{4} t_{2}\right)\right|,
\end{aligned}
$$

where $D(\phi)$ is the defect of $\phi$. From Lemmas 2.3, 2.5 and 2.6 we have

$$
D(\phi) \geq\left|\frac{1}{g} \phi\left(t_{1}\right)+(g+3) \phi\left(t_{1}\right)+g \phi\left(t_{1}\right)\right|=\left(2 g+3+\frac{1}{g}\right)\left|\phi\left(t_{1}\right)\right| .
$$
By Theorem 2.4 we have scl $\mathscr{H}_{g}\left(t_{1}\right) \leq \frac{1}{(2(2 g+3+1 / g))}$. This completes the proof
of Theorem 1.7. 
Remark 5.1. By a similar argument to the proof of Theorem 1.7, we can show that

$$
\operatorname{scl}_{\mathcal{M}_{0}^{m}}\left(\sigma_{1}\right)=\frac{1}{2\{m+1+2 /(m-2)\}} \text { for all } m \geq 4 .
$$

\section{Calculation of quasimorphisms}

In this section, we prove Theorem 1.1. To prove it, we perform a straightforward and elementary calculation of the hermitian form $\langle,\rangle_{\tilde{\sigma}^{k}, \tilde{\sigma}}$ on the eigenspace $V^{\omega^{j}}$.

Let $p: \Sigma_{g} \rightarrow S^{2}$ be the regular branched $m$-cyclic covering on $S^{2}$ with $m$ branched points as on page 333. Choose a point in $p^{-1}(*)$, and denote it by $\tilde{*} \in \Sigma_{g}$. We denote by $\tilde{\alpha}_{i}$ the lift of $\alpha_{i}$ which starts at $\tilde{*}$. Note that $\tilde{\alpha}_{i} t\left(\tilde{\alpha}_{i+1}\right)^{-1}$ is a loop in $\Sigma_{g}$ while $\tilde{\alpha}_{i}$ is an arc. We denote by $e_{i}(k) \in H_{1}\left(\Sigma_{g} ; \mathbb{Z}\right)$ the homology class represented by $t^{k}\left(\tilde{\alpha}_{i} t\left(\tilde{\alpha}_{i+1}\right)^{-1}\right)$.

Lemma 6.1. The homology classes $\left\{e_{i}(k)\right\}_{\substack{1 \leq i \leq m-2 \\ 0 \leq k \leq m-2}}$ form a basis of $H_{1}\left(\Sigma_{g} ; \mathbb{Z}\right)$.

Proof. We use the Schreier method. Let $T$ denote a Schreier transversal $T=\left\{\alpha_{1}^{k}\right\}_{i=0}^{m-1}$ and $S$ a generating set $S=\left\{\alpha_{i}\right\}_{i=1}^{m-1}$ of $\pi_{1}\left(S^{2}-\left\{q_{i}\right\}_{i=1}^{m}\right)$. Then the subgroup $\pi_{1}\left(\Sigma_{g}-\left\{p^{-1}\left(q_{i}\right)\right\}_{i=1}^{m}\right)$ is generated by

$$
\left\{\left(r s(\overline{r s})^{-1} \mid r \in T, s \in S\right\}=\left\{\alpha_{1}^{k} \alpha_{i} \alpha_{1}^{-k-1}\right\}_{\substack{2 \leq i \leq m-1 \\ 0 \leq k \leq m-2}} \cup\left\{\alpha_{1}^{m-1} \alpha_{i}\right\}_{1 \leq i \leq m-1} .\right.
$$

By van Kampen's theorem, the group $\pi_{1}\left(\Sigma_{g}\right)$ is obtained by adding the relation $\alpha_{i}^{m}=1$ to $\pi_{1}\left(\Sigma_{g}-\left\{p^{-1}\left(q_{i}\right)\right\}_{i=1}^{m}\right)$. Thus, the set $\left\{\alpha_{1}^{k} \alpha_{i} \alpha_{i+1}^{-1} \alpha_{1}^{-k}\right\}_{i, k}$, where from now through the end of the proof we have $1 \leq i \leq m-2$ and $0 \leq k \leq m-2$, generates the group $\pi_{1}\left(\Sigma_{g}\right)$. This implies that $\left\{e_{i}(k)\right\}_{i, k}$ is a generating set of $H_{1}\left(\Sigma_{g} ; \mathbb{Z}\right)$.

By the Riemann-Hurwitz formula, $H_{1}\left(\Sigma_{g} ; \mathbb{Z}\right)$ is a free module of rank equal to $2 g=(m-1)(m-2)$, and this is equal to the order of the set $\left\{e_{i}(k)\right\}_{i, k}$. Therefore the set $\left\{e_{i}(k)\right\}_{i, k}$ is a basis of the free module $H_{1}\left(\Sigma_{g} ; \mathbb{Z}\right)$.

The intersection form and the action of $\tilde{\sigma}_{i}$. Let $j$ be an integer with $1 \leq j \leq m-1$. Firstly we find a basis of $V^{\omega^{j}} \subset H_{1}\left(\Sigma_{g} ; \mathbb{C}\right)$ and calculate intersection numbers.

Lemma 6.2. The intersection numbers of $\left\{e_{i}(k)\right\}_{\substack{1 \leq i \leq m-2 \\ 0 \leq k \leq m-2}}$ are

$$
e_{i}(k) \cdot e_{i^{\prime}}(k)=\left\{\begin{array}{rl}
-1 & \text { if } i=i^{\prime}-1, \\
1 & \text { if } i=i^{\prime}+1, \\
0 & \text { otherwise, }
\end{array} \quad e_{i}(k) \cdot e_{i^{\prime}}(k+1)=\left\{\begin{aligned}
-1 & \text { if } i=i^{\prime}, \\
1 & \text { if } i=i^{\prime}-1, \\
0 & \text { otherwise, }
\end{aligned}\right.\right.
$$

$e_{i}(k) \cdot e_{i^{\prime}}(k-1)=\left\{\begin{array}{rl}-1 & \text { if } i=i^{\prime}, \\ 1 & \text { if } i=i^{\prime}+1, \\ 0 & \text { otherwise, }\end{array} \quad e_{i}(k) \cdot e_{i^{\prime}}\left(k^{\prime}\right)=0 \quad\right.$ if $\left|k-k^{\prime}\right| \geq 2$. 


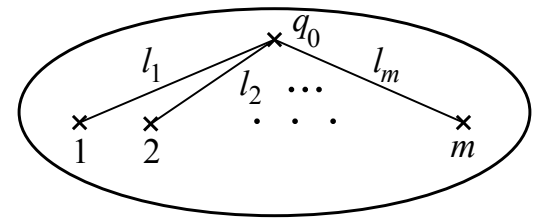

Figure 8. The paths $l_{1}, l_{2}, \ldots, l_{m}$.
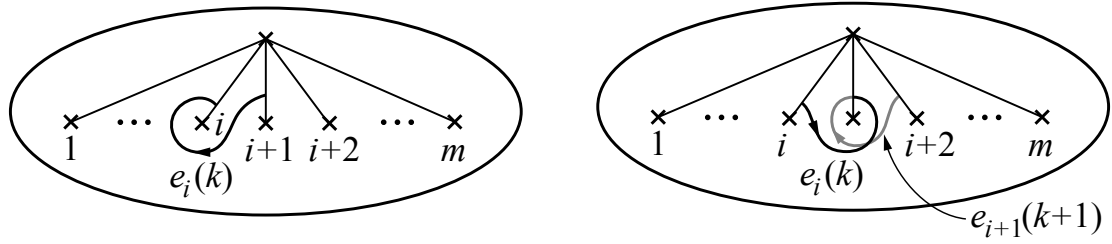

Figure 9. Left: the $k$-th copy. Right: the $(k+1)$-th copy.

Proof. We only prove the equality $e_{i}(k) \cdot e_{i+1}(k+1)=1$ since the other cases are proved in the same way.

Let $l_{i}$ be the paths as in Figure 8. Consider $m$ copies of the 2-sphere cut along the $l_{i}$, and number them from 1 to $m$. (For convenience, copy 1 will also be called copy $m+1$.) Gluing the left-hand side of $l_{i}$ in the $k$-th copy to the right-hand side of $l_{i}$ in the $(k+1)$-th copy for $k=1,2, \ldots, m$, we obtain a closed connected surface homeomorphic to $\Sigma_{g}$, and it is naturally a covering space on $S^{2}$. As in Figure 9, the loops representing $e_{i}(k)$ and $e_{i+1}(k+1)$ intersect once positively in the $(k+1)$-th copy.

Hence we have $e_{i}(k) \cdot e_{i+1}(k+1)=1$.

For $1 \leq i \leq m-2$, we define $w_{i}=\sum_{k=0}^{m-1} \omega^{-j k} e_{i}(k)$. Since $t e_{i}(k)=e_{i}(k+1)$ for $1 \leq k \leq m-2$ and $e_{i}(m-1)=-\sum_{k=0}^{m-2} e_{i}(k)$, we have $w_{i} \in V^{\omega^{j}}$, and the set $\left\{w_{i}\right\}_{i=1}^{m-2}$ is a basis of $V^{\omega^{j}}$.

Lemma 6.3. The intersection numbers of $\left\{w_{i}\right\}_{1 \leq i \leq m-2}$ are

$$
w_{i} \cdot w_{i^{\prime}}= \begin{cases}d\left(1-\omega^{j}\right) & \text { if } i=i^{\prime}+1, \\ d\left(-\omega^{-j}+\omega^{j}\right) & \text { if } i=i^{\prime}, \\ d\left(\omega^{-j}-1\right) & \text { if } i=i^{\prime}-1, \\ 0 & \text { otherwise. }\end{cases}
$$

Proof. By Lemma 6.2, we have

$$
\begin{aligned}
w_{i} \cdot w_{i} & =\sum_{k=0}^{d-1} \sum_{l=0}^{d-1} \omega^{j(k-l)} e_{i}(k) \cdot e_{i}(l)=d\left(-\omega^{-j}+\omega^{j}\right), \\
w_{i} \cdot w_{i+1} & =\sum_{k=0}^{d-1} \sum_{l=0}^{d-1} \omega^{j(k-l)} e_{i}(k) \cdot e_{i+1}(l)=d\left(\omega^{-j}-1\right),
\end{aligned}
$$


and $w_{i} \cdot w_{k}=0$ when $|i-k| \geq 2$.

Let $\tilde{\sigma}=\tilde{\sigma}_{1} \cdots \tilde{\sigma}_{r-1}$. We next find eigenvectors in $V^{\omega^{j}}$ relative to the action by $\tilde{\sigma}$. Lemma 6.4. Let $i$ be an integer such that $1 \leq i \leq m-1$. Then we have

$$
\left(\tilde{\sigma}_{i}\right)_{*} e_{l}(k)= \begin{cases}e_{l}(k)+e_{l+1}(k) & \text { if } 2 \leq i \leq m-1, \text { and } l=i-1, \\ -e_{l}(k-1) & \text { if } l=i, \\ e_{l-1}(k-1)+e_{l}(k) & \text { if } l=i+1, \\ e_{l}(k) & \text { if } l \neq i-1, i, i+1 .\end{cases}
$$

Proof. Recall that $e_{i}(k)$ is the homology class represented by the loop $\tilde{\alpha}_{1}^{k} \tilde{\alpha}_{i} \tilde{\alpha}_{i+1}^{-1} \tilde{\alpha}_{1}^{-k}$. In the fundamental group $\pi_{1}\left(S^{2}-\left\{q_{i}\right\}_{i=1}^{m}\right)$, we have

$$
\begin{aligned}
\left(\sigma_{i}\right)_{*}\left(\alpha_{i-1} \alpha_{i}^{-1}\right) & =\alpha_{i-1} \alpha_{i+1}^{-1}=\left(\alpha_{i-1} \alpha_{i}^{-1}\right)\left(\alpha_{i} \alpha_{i+1}^{-1}\right), \\
\left(\sigma_{i}\right)_{*}\left(\alpha_{i} \alpha_{i+1}^{-1}\right) & =\alpha_{i+1}\left(\alpha_{i+1}^{-1} \alpha_{i} \alpha_{i+1}\right)^{-1}=\alpha_{i+1}^{-1}\left(\alpha_{i} \alpha_{i+1}^{-1}\right)^{-1} \alpha_{i+1}, \\
\left(\sigma_{i}\right)_{*}\left(\alpha_{i+1} \alpha_{i+2}^{-1}\right) & =\left(\alpha_{i+1}^{-1} \alpha_{i} \alpha_{i+1}\right) \alpha_{i+2}^{-1}=\alpha_{i+1}^{-1}\left(\alpha_{i} \alpha_{i+1}^{-1}\right) \alpha_{i+1}\left(\alpha_{i+1} \alpha_{i+2}^{-1}\right) .
\end{aligned}
$$

By lifting these loops to the covering space $\Sigma_{g}$, we obtain what we want.

By Lemma 6.4, the matrix representations of the actions of $\left\{\tilde{\sigma}_{i}\right\}_{i=1}^{m-1}$ on $V^{\omega^{j}}$ with respect to the basis $\left\{w_{i}\right\}_{1 \leq i \leq m-2}$ are calculated as

$$
\begin{array}{rlr}
\left(\tilde{\sigma}_{1}\right)_{*}=\left(\begin{array}{ccc}
-\omega^{-j} & \omega^{-j} & O \\
0 & 1 & O \\
O & O & I_{m-4}
\end{array}\right), & \left(\tilde{\sigma}_{i}\right)_{*}=\left(\begin{array}{ccc}
I_{i-1} & O & O \\
O & L & O \\
O & O & I_{m-i-4}
\end{array}\right), \\
\left(\tilde{\sigma}_{m-2}\right)_{*}=\left(\begin{array}{ccc}
I_{m-4} & O & O \\
O & 1 & O \\
O & 1 & -\omega^{-j}
\end{array}\right), & \left(\tilde{\sigma}_{m-1}\right)_{*}=\left(\begin{array}{cc}
I_{m-3} & v \\
O & -1+\sum_{k=1}^{m-2} \omega^{-j k}
\end{array}\right),
\end{array}
$$

where

$$
L=\left(\begin{array}{ccc}
1 & 0 & 0 \\
1 & -\omega^{-j} & \omega^{-j} \\
0 & 0 & 1
\end{array}\right), \quad v=\left(1,1+\omega^{-j}, \ldots, \sum_{k=0}^{m-3} \omega^{-j k}\right)^{T}
$$

Let $r$ be an integer with $2 \leq r \leq m$, and put

$$
e_{r}^{\prime}(k)=\left[\tilde{a}_{1}^{k} \tilde{a}_{r}\left(\tilde{a}_{1} \tilde{a}_{2} \cdots \tilde{a}_{r}\right)^{-1} \tilde{a}_{1}^{-1}\left(\tilde{a}_{1} \tilde{a}_{2} \cdots \tilde{a}_{r}\right) \tilde{a}_{1}^{-k}\right] .
$$

By Lemma 6.4, we have

$$
\begin{aligned}
\tilde{\sigma}_{*} e_{i}(k) & =e_{i+1}(k), \quad \text { when } 1 \leq i \leq r-2, \\
\tilde{\sigma}_{*} e_{r}(k) & =-e_{r}^{\prime}(k)+e_{r}(k), \\
\tilde{\sigma}_{*} e_{r-1}(k) & =e_{r}^{\prime}(k), \\
\tilde{\sigma}_{*} e_{r}^{\prime}(k) & =e_{1}(k-r+1) .
\end{aligned}
$$


The sum $w_{r}^{\prime}:=\sum_{k=0}^{m-1} \omega^{-j k} e_{r}^{\prime}(k)$ is contained in $V^{\omega^{j}}$. For $i=1,2, \ldots, r-2$, we have

$$
\begin{aligned}
\tilde{\sigma}_{*} w_{i} & =\tilde{\sigma}_{*} \sum_{k=0}^{m-1} \omega^{-j k} e_{i}(k)=\sum_{k=0}^{m-1} \omega^{-j k} e_{i+1}(k)=w_{i+1}, \\
\tilde{\sigma}_{*} w_{r-1} & =\sum_{k=0}^{m-1} \omega^{-j k}\left(e_{r-1}(k)+e_{r}^{\prime}(k)\right)=w_{r-1}+w_{r}^{\prime}, \\
\tilde{\sigma}_{*} w_{r}^{\prime} & =\sum_{k=0}^{m-1} \omega^{-j k} e_{1}(k-r+1)=\sum_{k=0}^{m-1} \omega^{-j(k+r-1)} e_{1}(k)=\omega^{-(r-1) j} w_{1} .
\end{aligned}
$$

Let $\zeta=\exp (2 \pi i / r)$ and $v_{i}=\sum_{k=1}^{r-1} \omega^{(k-1) j} \zeta^{-(k-1) i} w_{k}+\omega^{(r-1) j} \zeta^{-(r-1) i} w_{r}^{\prime}$. Then

$$
\begin{aligned}
\tilde{\sigma}_{*} v_{i} & =\sum_{k=1}^{r-1} \omega^{(k-1) j} \zeta^{-(k-1) i}(\tilde{\sigma})_{*} w_{k}+\omega^{(r-1) j} \zeta^{-(r-1) i}(\tilde{\sigma})_{*} w_{r}^{\prime} \\
& =\sum_{k=1}^{r-2} \omega^{(k-1) j} \zeta^{-(k-1) i} w_{k+1}+\omega^{(r-2) j} \zeta^{-(r-2) i} w_{r}^{\prime}+\omega^{(r-1) j} \zeta^{-(r-1) i} \omega^{-p j} w_{1} \\
& =\omega^{-j} \zeta^{i}\left(\sum_{k=1}^{r-1} \omega^{(k-1) j} \zeta^{-(k-1) i} w_{k}+\omega^{(r-1) j} \zeta^{-(r-1) i} w_{r}^{\prime}\right) \\
& =\left(\omega^{-j} \zeta^{i}\right) v_{i} .
\end{aligned}
$$

Hence $v_{i}$ is an eigenvector with eigenvalue $\omega^{-j} \zeta^{i}$ with respect to the action by $\tilde{\sigma}$. Note that the subspace generated by $\left\{w_{i}\right\}_{i=1}^{r-1}$ coincides with one generated by $\left\{v_{i}\right\}_{i=1}^{r-1}$. Since $\tilde{\sigma}$ acts trivially on $\left\{w_{i}\right\}_{i=r+1}^{m-1}$, they are also eigenvectors with eigenvalue 0 . Moreover the set $\left\{v_{i}\right\}_{i=1}^{r-1} \cup\left\{w_{i}\right\}_{i=r+1}^{m-2}$ is linearly independent.

Lemma 6.5. Let $i$, $i^{\prime}$ be integers such that $1 \leq i \leq r-1$ and $1 \leq i^{\prime} \leq r-1$. Then we have

$$
v_{i} \cdot v_{i^{\prime}}= \begin{cases}8 r d i \sin \frac{\pi i}{r} \sin \frac{\pi j}{m} \sin \pi\left(\frac{i}{r}-\frac{j}{m}\right) & \text { if } i=i^{\prime} \\ 0 & \text { otherwise. }\end{cases}
$$

Proof. Since the action of the mapping class group $\pi_{0} C_{g}(t)$ preserves the intersection form,

$$
\begin{aligned}
v_{i} \cdot v_{i} & =\sum_{k=0}^{r-1} \sum_{l=0}^{r-1} \omega^{(l-k) j} \zeta^{-(l-k) i}\left(\tilde{\sigma}_{*}^{k} w_{1} \cdot \tilde{\sigma}_{*}^{l} w_{1}\right) \\
& =\sum_{k=0}^{r-1} \sum_{l=0}^{r-1} \omega^{(l-k) j} \zeta^{-(l-k) i}\left(w_{2} \cdot \tilde{\sigma}_{*}^{l-k+1} w_{1}\right) .
\end{aligned}
$$


Thus Lemma 6.3 implies

$$
\begin{aligned}
v_{i} \cdot v_{i}= & \omega^{(r-1) j} \zeta^{-(r-1) i}\left(w_{2} \cdot \tilde{\sigma}_{*}^{r} w_{1}\right)+\omega^{j} \zeta^{-i}(r-1)\left(w_{2} \cdot \tilde{\sigma}_{*}^{2} w_{1}\right)+r\left(w_{2} \cdot \tilde{\sigma}_{*} w_{1}\right) \\
& \quad+\omega^{-j} \zeta^{i}(r-1)\left(w_{2} \cdot w_{1}\right)+\omega^{-(r-1) j} \zeta^{(r-1) i}\left(w_{2} \cdot \tilde{\sigma}_{*}^{-r+2} w_{1}\right) \\
= & r\left\{\left(\omega^{-j} \zeta^{i}\right) w_{2} \cdot w_{1}+\left(\omega^{j} \zeta^{-i}\right) w_{2} \cdot w_{3}+w_{2} \cdot w_{2}\right\} \\
= & 8 r d i \sin \frac{\pi i}{r} \sin \frac{\pi j}{m} \sin \pi\left(\frac{i}{r}-\frac{j}{m}\right) .
\end{aligned}
$$

Calculation of $\omega$-signatures and the cobounding functions $\phi_{m, j}$. Lastly we will calculate the hermitian form $\langle,\rangle_{\tilde{\sigma}^{k}, \tilde{\sigma}}$ and the $\omega$-signature. We have already found the set of eigenvectors $\left\{v_{i}\right\}_{i=1}^{r-1} \cup\left\{w_{i}\right\}_{i=r+1}^{m-2}$ with respect to the action by $\tilde{\sigma}$ which is linearly independent. Since $\operatorname{dim} V^{\omega^{j}}=m-2$, we need to find another eigenvector.

Lemma 6.6.

$$
\sum_{k=1}^{r m} \tau\left(\tilde{\sigma}^{k}, \tilde{\sigma}\right)=r m-2|m i-r j| .
$$

Proof. We first consider the case when $r j / m$ is not an integer. Put

$$
\beta=\sum_{i=1}^{r} w_{i}-\frac{1}{r} \sum_{k=1}^{r} \frac{1}{1-\omega^{j} \zeta^{-k}} v_{k} .
$$

The subspace generated by $\left\{v_{i}\right\}_{i=1}^{r-1}$ and that generated by $\left\{w_{i}\right\}_{i=1}^{r-1}$ coincide. Thus the set $\left\{v_{i}\right\}_{i=1}^{r-1}, \beta,\left\{w_{i}\right\}_{i=r+1}^{m-2}$ forms a basis of $V^{\omega^{j}}$ when $1 \leq r \leq m-2$, and the set $\left\{v_{i}\right\}_{i=1}^{m-2}$ forms a basis of $V^{\omega^{j}}$ when $r=m-1$. We have

$$
\begin{aligned}
\tilde{\sigma}_{*} \beta & =\sum_{i=2}^{r} w_{i}-\frac{1}{r} \sum_{k=1}^{r} \frac{\omega^{j} \zeta^{-k}}{1-\omega^{j} \zeta^{-k}} v_{k} \\
& =\sum_{i=2}^{r} w_{i}+\frac{1}{r} \sum_{k=1}^{r} v_{k}-\frac{1}{r} \sum_{k=1}^{r} \frac{1}{1-\omega^{j} \zeta^{-k}} v_{k} \\
& =\sum_{i=1}^{r} w_{i}-\frac{1}{r} \sum_{k=1}^{r} \frac{1}{1-\omega^{j} \zeta^{-k}} v_{k}=\beta .
\end{aligned}
$$

Note that $\beta$ and $\left\{w_{i}\right\}_{i=r+1}^{m-2}$ are in the annihilator of the hermitian form $\langle,\rangle_{\tilde{\sigma}^{k}, \tilde{\sigma}}$ since they have eigenvalue 1 with respect to the action by $\tilde{\sigma}$.

By Lemma 4.2, we have

$$
\begin{aligned}
\tau\left(\tilde{\sigma}^{k}, \tilde{\sigma}\right) & =\sum_{i=1}^{r} \operatorname{sign}\left\langle v_{i}, v_{i}\right\rangle_{\tilde{\sigma}^{k}, \tilde{\sigma}}=-\sum_{i=1}^{r} \operatorname{sign}\left(\left(v_{i} \cdot v_{i}\right) \sum_{l=1}^{k}\left(\left(\omega^{-j} \zeta^{i}\right)^{l}-\left(\omega^{j} \zeta^{-i}\right)^{l}\right)\right) \\
& =-\sum_{i=1}^{r} \operatorname{sign}\left(\left(v_{i} \cdot v_{i}\right)\left(1-\omega^{j} \zeta^{-i}\right)\left(1-\omega^{-j} \zeta^{i}\right) \sum_{l=1}^{k}\left(\left(\omega^{-j} \zeta^{i}\right)^{l}-\left(\omega^{j} \zeta^{-i}\right)^{l}\right)\right)
\end{aligned}
$$


By the equation

$$
\begin{aligned}
& \left(1-\omega^{j} \zeta^{-i}\right)\left(1-\omega^{-j} \zeta^{i}\right) \sum_{l=1}^{k}\left(\left(\omega^{-j} \zeta^{i}\right)^{l}-\left(\omega^{j} \zeta^{-i}\right)^{l}\right) \\
& \quad=8 \boldsymbol{i} \sin \left(-\frac{\pi(k+1) j}{m}+\frac{\pi(k+1) i}{r}\right) \sin \left(-\frac{\pi k j}{m}+\frac{\pi k i}{r}\right) \sin \left(-\frac{\pi j}{m}+\frac{\pi i}{r}\right)
\end{aligned}
$$

and Lemma 6.5, we have

$$
\tau\left((\tilde{\sigma})^{k}, \tilde{\sigma}\right)=\sum_{i=1}^{r-1} \operatorname{sign}\left(\sin k \pi\left(\frac{i}{r}-\frac{j}{m}\right) \sin (k+1) \pi\left(\frac{i}{r}-\frac{j}{m}\right)\right) .
$$

Since $r j / m$ is not an integer, $i / r-j / m$ is not zero. Thus we obtain

$$
\begin{aligned}
\sum_{k=1}^{r m} \tau\left((\tilde{\sigma})^{k}, \tilde{\sigma}\right) & =\sum_{i=1}^{r-1} \sum_{k=1}^{r m} \operatorname{sign}\left(\sin k \pi\left(\frac{i}{r}-\frac{j}{m}\right) \sin (k+1) \pi\left(\frac{i}{r}-\frac{j}{m}\right)\right) \\
& =\sum_{i=1}^{r-1}(r m-2|m i-r j|) .
\end{aligned}
$$

Next consider the case when $r j / m$ is an integer and $1 \leq r \leq m-1$. Denote this integer $r j / m$ by $i_{0}$. Then, the eigenvalue of $v_{i_{0}}$ is 1 , and $v_{i_{0}}$ and $\left\{w_{i}\right\}_{i=r+1}^{m-2}$ are in the annihilator of $\langle,\rangle_{\tilde{\sigma}^{k}, \tilde{\sigma}}$. If we put

$$
\beta^{\prime}=\sum_{i=1}^{r} w_{i}-\frac{1}{r} \sum_{\substack{1 \leq k \leq r \\ k \neq i_{0}}} \frac{1}{1-\omega^{j} \zeta^{-k}} v_{k}
$$

the set of the homology classes $\left\{v_{i}\right\}_{i=1}^{r-1}, \beta^{\prime},\left\{w_{i}\right\}_{i=r+1}^{m-2}$ forms a basis of $V^{\omega^{j}}$. We have

$$
\begin{aligned}
\tilde{\sigma} \beta^{\prime} & =\sum_{i=2}^{r} w_{i}-\frac{1}{r} \sum_{\substack{1 \leq k \leq r \\
k \neq i_{0}}} \frac{\omega^{j} \zeta^{-k}}{1-\omega^{j} \zeta^{-k}} v_{k} \\
& =\sum_{i=2}^{r} w_{i}+\frac{1}{r} \sum_{\substack{1 \leq k \leq r \\
k \neq i_{0}}} v_{k}-\frac{1}{r} \sum_{\substack{1 \leq k \leq r \\
k \neq i_{0}}} \frac{1}{1-\omega^{j} \zeta^{-k}} v_{k} \\
& =\sum_{i=1}^{r} w_{i}-\frac{1}{r} v_{i_{0}}-\frac{1}{r} \sum_{\substack{1 \leq k \leq r \\
k \neq i_{0}}} \frac{1}{1-\omega^{j} \zeta^{-k}} v_{k}=\beta^{\prime}-\frac{1}{r} v_{i_{0}} .
\end{aligned}
$$

By Lemma 4.2,

$$
\left\langle\beta^{\prime}, \beta^{\prime}\right\rangle_{\tilde{\sigma}^{k}, \tilde{\sigma}}=\beta^{\prime} \cdot \frac{1}{r} \sum_{i=1}^{k} 2 i v_{i_{0}}=\frac{k(k+1)}{r} \sum_{i=1}^{r} w_{i} \cdot v_{i_{0}} .
$$


Since the eigenvalues of $\left\{v_{i}\right\}_{i=1}^{r-1}$ are different from 1 , the intersection $v_{i} \cdot v_{i_{0}}=0$ for $1 \leq i \leq r-1$. Since the subspace generated by $\left\{w_{i}\right\}_{i=1}^{r-1}$ and that generated by $\left\{v_{i}\right\}_{i=1}^{r-1}$ coincide, we also have $w_{i} \cdot v_{i_{0}}=0$. Thus we have

$$
\begin{aligned}
\frac{r}{k(k+1)}\left\langle\beta^{\prime}, \beta^{\prime}\right\rangle_{\tilde{\sigma}^{k}, \tilde{\sigma}} & =w_{r} \cdot v_{i_{0}}=w_{r} \cdot\left(w_{r-1}+w_{r}^{\prime}\right) \\
& =w_{r} \cdot\left(w_{r-1}-\sum_{k=0}^{r-1} \omega^{(k-r) j} w_{k}\right) \\
& =\left(1-\omega^{-j}\right) w_{r} \cdot w_{r-1}=\left(1-\omega^{-j}\right)\left(1-\omega^{j}\right)>0 .
\end{aligned}
$$

Moreover since $v_{i} \cdot v_{i_{0}}=0$, Lemma 4.2 implies $\left\langle v_{i}, \beta^{\prime}\right\rangle_{\tilde{\sigma}^{k}, \tilde{\sigma}}=0$ for $1 \leq i \leq r-1$. Therefore we have

$$
\begin{aligned}
\sum_{k=1}^{r m} \tau\left(\tilde{\sigma}^{k}, \tilde{\sigma}\right) & =\sum_{k=1}^{r m}\left(\sum_{i=1}^{k} \operatorname{sign}\left(\left\langle v_{i}, v_{i}\right\rangle_{\tilde{\sigma}^{k}, \tilde{\sigma}}\right)+\operatorname{sign}\left(\left\langle\beta^{\prime}, \beta^{\prime}\right\rangle_{\tilde{\sigma}^{k}, \tilde{\sigma}}\right)\right) \\
& =\sum_{k=1}^{r m}\left(\sum_{\substack{1 \leq i \leq r-1 \\
i \neq i_{0}}} \operatorname{sign}\left(\sin k \pi\left(\frac{i}{r}-\frac{j}{m}\right) \sin (k+1) \pi\left(\frac{i}{r}-\frac{j}{m}\right)\right)+1\right) \\
& =\sum_{\substack{1 \leq i \leq r-1 \\
i \neq i_{0}}}(r m-2|m i-r j|)+r m=\sum_{i=1}^{r-1}(r m-2|m i-r j|) .
\end{aligned}
$$

In the case when $r=m$, the set $\left\{v_{i}\right\}_{i=1}^{r-2}$ forms a basis of $V^{\omega^{j}}$. By a similar calculation, we can also prove what we want.

Lemma 6.7. For $r=2,3, \ldots, m$,

$$
\begin{aligned}
& \qquad \phi_{m, j}(\tilde{\sigma})-\bar{\phi}_{m, j}(\tilde{\sigma})=\frac{2}{r}\left\{\left(\frac{r j}{m}-\left[\frac{r j}{m}\right]-\frac{1}{2}\right)^{2}-\frac{r^{2} j(m-j)}{m^{2}}-\frac{1}{4}\right\} . \\
& \text { Proof. } \quad \tau\left(\tilde{\sigma}^{k}, \tilde{\sigma}\right)=\sum_{i=1}^{r-1} \operatorname{sign}\left(\sin k \pi\left(\frac{i}{r}-\frac{j}{m}\right) \sin (k+1) \pi\left(\frac{i}{r}-\frac{j}{m}\right)\right) .
\end{aligned}
$$

Since we have $\tau\left(\tilde{\sigma}^{k+r m}, \tilde{\sigma}\right)=\tau\left(\tilde{\sigma}^{k}, \tilde{\sigma}\right)$,

$$
\begin{aligned}
\phi_{m, j}(\tilde{\sigma})-\bar{\phi}_{m, j}(\tilde{\sigma}) & =\frac{1}{r m} \sum_{k=1}^{r m} \tau\left(\tilde{\sigma}^{k}, \tilde{\sigma}\right)=\frac{1}{r m} \sum_{i=1}^{r-1}(r m-2|m i-r j|) \\
& =r-1-\frac{2}{r m}\left(\sum_{i=1}^{\left[\frac{r j}{m}\right]}(r j-m i)+\sum_{\left[\frac{r j}{m}\right]+1}^{r-1}(m i-r j)\right) \\
& =\frac{2}{r}\left\{\left(\frac{r j}{m}-\left[\frac{r j}{m}\right]-\frac{1}{2}\right)^{2}+\frac{r^{2} j(m-j)}{m^{2}}-\frac{1}{4}\right\} .
\end{aligned}
$$


Proof of Theorem 1.1. Applying Lemma 6.7 to the case when $r=m$, we have

$$
\phi_{m, j}\left(\tilde{\sigma}_{1} \cdots \tilde{\sigma}_{m-1}\right)-\bar{\phi}_{m, j}\left(\tilde{\sigma}_{1} \cdots \tilde{\sigma}_{m-1}\right)=\frac{2 j(m-j)}{m} .
$$

Since

$$
\bar{\phi}_{m, j}\left(\tilde{\sigma}_{1} \cdots \tilde{\sigma}_{m-1}\right)=\frac{1}{m} \bar{\phi}_{m, j}\left(\left(\tilde{\sigma}_{1} \cdots \tilde{\sigma}_{m-1}\right)^{m}\right)=0,
$$

we have

$$
\phi_{m, j}\left(\tilde{\sigma}_{1} \cdots \tilde{\sigma}_{m-1}\right)=\frac{2 j(m-j)}{m} .
$$

Put $\varphi=\tilde{\sigma}_{1} \tilde{\sigma}_{3} \cdots \tilde{\sigma}_{m-1}, \psi=\tilde{\sigma}_{2} \tilde{\sigma}_{4} \cdots \tilde{\sigma}_{m-2}$ when $m$ is even, and $\varphi=\tilde{\sigma}_{1} \tilde{\sigma}_{3} \cdots \tilde{\sigma}_{m-2}$, $\psi=\tilde{\sigma}_{2} \tilde{\sigma}_{4} \cdots \tilde{\sigma}_{m-1}$, when $m$ is odd. As we saw in Section $5, \tilde{\sigma}_{1} \cdots \tilde{\sigma}_{m-1}$ is conjugate to $\varphi \psi$. By direct computation, if $\left(\varphi_{*}^{-1}-I_{2 g}\right) x+\left(\psi_{*}-I_{2 g}\right) y=0$ for $x, y \in V^{\omega^{j}}$, we have $\left(\varphi_{*}^{-1}-I_{2 g}\right) x=\left(\psi_{*}-I_{2 g}\right) y=0$. Hence we have $\tau_{g}(\varphi, \psi)=0$.

In the same way, for $i=1,2, \ldots,[(m-1) / 2]$, we have

$$
\begin{gathered}
\tau_{g}\left(\tilde{\sigma}_{1} \tilde{\sigma}_{3} \cdots \tilde{\sigma}_{2 i+1}, \tilde{\sigma}_{2} \tilde{\sigma}_{4} \cdots \tilde{\sigma}_{2 i}\right)=\tau_{g}\left(\tilde{\sigma}_{1} \tilde{\sigma}_{3} \cdots \tilde{\sigma}_{2 i+1}, \tilde{\sigma}_{2} \tilde{\sigma}_{4} \cdots \tilde{\sigma}_{2 i+2}\right)=0 \\
\tau_{g}\left(\tilde{\sigma}_{1} \tilde{\sigma}_{3} \cdots \tilde{\sigma}_{2 i-1}, \tilde{\sigma}_{2 i+1}\right)=\tau_{g}\left(\tilde{\sigma}_{2} \tilde{\sigma}_{4} \cdots \tilde{\sigma}_{2 i}, \tilde{\sigma}_{2 i+2}\right)=0 .
\end{gathered}
$$

Thus

$$
\phi_{m, j}(\tilde{\sigma})=(r-1) \phi_{m, j}\left(\tilde{\sigma}_{1}\right)=\frac{r-1}{m-1} \phi_{m, j}\left(\tilde{\sigma}_{1} \cdots \tilde{\sigma}_{m-1}\right)=\frac{2(r-1) j(m-j)}{m(m-1)} .
$$

Hence we obtain

$$
\begin{aligned}
\bar{\phi}_{m, j}\left(\sigma_{1} \cdots \sigma_{r-1}\right) & =\bar{\phi}_{m, j}(\tilde{\sigma}) \\
& =\phi_{m, j}(\tilde{\sigma})-\left(\phi_{m, j}(\tilde{\sigma})-\bar{\phi}_{m, j}(\tilde{\sigma})\right) \\
& =-\frac{2}{r}\left\{\frac{j r(m-j)(m-r)}{m^{2}(m-1)}+\left(\frac{r j}{m}-\left[\frac{r j}{m}\right]-\frac{1}{2}\right)^{2}-\frac{1}{4}\right\} .
\end{aligned}
$$

By the values of $\bar{\phi}_{m, 1}$, we see:

Remark 6.8. Let $r$ be an integer such that $2 \leq r \leq m$. Then

$$
\bar{\phi}_{m, 1}\left(\tilde{\sigma}_{1} \cdots \tilde{\sigma}_{r-1}\right)=0 .
$$

However we do not know whether the quasimorphism $\bar{\phi}_{m, 1}$ is trivial or not.

\section{References}

[Barge and Ghys 1992] J. Barge and É. Ghys, "Cocycles d'Euler et de Maslov", Math. Ann. 294:2 (1992), 235-265. MR 95b:55021 Zbl 0894.55006

[Bavard 1991] C. Bavard, "Longueur stable des commutateurs", Enseign. Math. (2) 37:1-2 (1991), 109-150. MR 92g:20051 Zbl 0810.20026 
[Baykur et al. 2013] R. İ. Baykur, M. Korkmaz, and N. Monden, "Sections of surface bundles and Lefschetz fibrations", Trans. Amer. Math. Soc. 365:11 (2013), 5999-6016. MR 3091273 Zbl 06208247

[Bestvina and Fujiwara 2002] M. Bestvina and K. Fujiwara, "Bounded cohomology of subgroups of mapping class groups”, Geom. Topol. 6 (2002), 69-89. MR 2003f:57003 Zbl 1021.57001

[Birman and Hilden 1971] J. S. Birman and H. M. Hilden, "On the mapping class groups of closed surfaces as covering spaces", pp. 81-115 in Advances in the theory of Riemann surfaces (Stony Brook, NY, 1969), edited by L. V. Ahlfors et al., Ann. of Math. Studies 66, Princeton University Press, 1971. MR 45 \#1169 Zbl 0217.48602

[Birman and Hilden 1973] J. S. Birman and H. M. Hilden, "On isotopies of homeomorphisms of Riemann surfaces”, Ann. of Math. (2) 97 (1973), 424-439. MR 48 \#4305 Zbl 0237.57001

[Calegari 2009] D. Calegari, scl, MSJ Memoirs 20, Math. Soc. Japan, Tokyo, 2009. MR 2011b:57003 Zbl 1187.20035

[Cochran et al. 2012] T. D. Cochran, S. Harvey, and P. D. Horn, "Higher-order signature cocycles for subgroups of mapping class groups and homology cylinders", Int. Math. Res. Not. 2012:14 (2012), 3311-3373. MR 2946227 Zbl 1257.57014

[Cohen 1987] F. R. Cohen, "Homology of mapping class groups for surfaces of low genus", pp. 21-30 in The Lefschetz Centennial Conference (Mexico City, 1984), edited by S. Gitler, Contemp. Math. 58:2, Amer. Math. Soc., Providence, RI, 1987. MR 88j:57046 Zbl 0655.57006

[Endo 2000] H. Endo, "Meyer's signature cocycle and hyperelliptic fibrations", Math. Ann. 316:2 (2000), 237-257. MR 2001b:57047 Zbl 0948.57013

[Endo and Kotschick 2001] H. Endo and D. Kotschick, "Bounded cohomology and non-uniform perfection of mapping class groups", Invent. Math. 144:1 (2001), 169-175. MR 2001m:57046 Zbl 0987.57004

[Endo and Nagami 2005] H. Endo and S. Nagami, "Signature of relations in mapping class groups and non-holomorphic Lefschetz fibrations", Trans. Amer. Math. Soc. 357:8 (2005), 3179-3199. MR 2006g:57051 Zbl 1081.57023

[Farb and Margalit 2012] B. Farb and D. Margalit, A primer on mapping class groups, Princeton Mathematical Series 49, Princeton University Press, 2012. MR 2012h:57032 Zbl 1245.57002

[Gambaudo and Ghys 2005] J.-M. Gambaudo and É. Ghys, "Braids and signatures", Bull. Soc. Math. France 133:4 (2005), 541-579. MR 2007d:57014 Zbl 1103.57001

[Korkmaz 2004] M. Korkmaz, "Stable commutator length of a Dehn twist", Michigan Math. J. 52:1 (2004), 23-31. MR 2005b:57003 Zbl 1061.57022

[Kotschick 2008] D. Kotschick, "Stable length in stable groups", pp. 401-413 in Groups of diffeomorphisms, edited by R. Penner et al., Adv. Stud. Pure Math. 52, Math. Soc. Japan, Tokyo, 2008. MR 2011j:57003 Zbl 1188.20028

[Louwsma 2011] J. R. Louwsma, Extremality of the rotation quasimorphism on the modular group, Ph.D. thesis, California Institute of Technology, Pasadena, CA, 2011, Available at http:// search.proquest.com/docview/1022051368. MR 3047198

[Meyer 1972] W. Meyer, "Die Signatur von lokalen Koeffizientensystemen und Faserbündeln", Bonn. Math. Schr. 53 (1972). MR 46 \#4532 Zbl 0243.58004

[Monden 2012] N. Monden, "On upper bounds on stable commutator lengths in mapping class groups”, Topology Appl. 159:4 (2012), 1085-1091. MR 2876715 Zbl 1239.57035

[Morifuji 2003] T. Morifuji, "On Meyer's function of hyperelliptic mapping class groups", J. Math. Soc. Japan 55:1 (2003), 117-129. MR 2003m:57054 Zbl 1031.57017 
[Powell 1978] J. Powell, "Two theorems on the mapping class group of a surface", Proc. Amer. Math. Soc. 68:3 (1978), 347-350. MR 58 \#13045 Zbl 0391.57009

[Turaev 1985] V. G. Turaev, "Первый симплектический класс Черна и индексы Маслова”, Zap. Nauchn. Sem. Leningrad. Otdel. Mat. Inst. Steklov. (LOMI) 143 (1985), 110-129. Translated as "First symplectic Chern class and Maslov indices" in J. Sov. Math. 37:3 (1987), 1115-1127. MR 86m:58059 Zbl 0577.55012

Received August 23, 2013. Revised February 28, 2014.

DANNY CALEGARI

DEPARTMENT OF MATHEMATICS

UNIVERSITY OF CHICAGO

5734 South University AVEnue

Chicago, IL 60637

UNITED STATES

dannyc@math.uchicago.edu

NAOYUKI MONDEN

DEPARTMENT OF ENGINEERING SCIENCE

OSAKA ELECTRO-COMMUNICATION UNIVERSITY

HATSU-CHO 18-8

NEYAGAWA 572-8530

JAPAN

n-monden@math.kyoto-u.ac.jp

Masatoshi SATO

DEPARTMENT OF MATHEMATICS EdUCATION

GIFU UNIVERSITY

YANAGITO 1-1

GIFU 501-1193

JAPAN

msato@gifu-u.ac.jp 


\title{
PACIFIC JOURNAL OF MATHEMATICS
}

\author{
msp.org/pjm
}

Founded in 1951 by E. F. Beckenbach (1906-1982) and F. Wolf (1904-1989)

\section{EDITORS}

Don Blasius (Managing Editor)

Department of Mathematics

University of California

Los Angeles, CA 90095-1555

blasius@math.ucla.edu

\author{
Paul Balmer \\ Department of Mathematics \\ University of California \\ Los Angeles, CA 90095-1555 \\ balmer@math.ucla.edu \\ Robert Finn \\ Department of Mathematics \\ Stanford University \\ Stanford, CA 94305-2125 \\ finn@math.stanford.edu \\ Sorin Popa \\ Department of Mathematics \\ University of California \\ Los Angeles, CA 90095-1555 \\ popa@math.ucla.edu
}

\author{
Vyjayanthi Chari \\ Department of Mathematics \\ University of California \\ Riverside, CA 92521-0135 \\ chari@math.ucr.edu \\ Kefeng Liu \\ Department of Mathematics \\ University of California \\ Los Angeles, CA 90095-1555 \\ liu@math.ucla.edu \\ Jie Qing \\ Department of Mathematics \\ University of California \\ Santa Cruz, CA 95064 \\ qing@ cats.ucsc.edu
}

\section{PRODUCTION}

Silvio Levy, Scientific Editor, production@msp.org

\section{SUPPORTING INSTITUTIONS}

ACADEMIA SINICA, TAIPEI

CALIFORNIA INST. OF TECHNOLOGY

INST. DE MATEMÁTICA PURA E APLICADA

KEIO UNIVERSITY

MATH. SCIENCES RESEARCH INSTITUTE

NEW MEXICO STATE UNIV.

OREGON STATE UNIV.

\author{
STANFORD UNIVERSITY \\ UNIV. OF BRITISH COLUMBIA \\ UNIV. OF CALIFORNIA, BERKELEY \\ UNIV. OF CALIFORNIA, DAVIS \\ UNIV. OF CALIFORNIA, LOS ANGELES \\ UNIV. OF CALIFORNIA, RIVERSIDE \\ UNIV. OF CALIFORNIA, SAN DIEGO \\ UNIV. OF CALIF., SANTA BARBARA
}

\author{
Daryl Cooper \\ Department of Mathematics \\ University of California \\ Santa Barbara, CA 93106-3080 \\ cooper@math.ucsb.edu \\ Jiang-Hua Lu \\ Department of Mathematics \\ The University of Hong Kong \\ Pokfulam Rd., Hong Kong \\ jhlu@maths.hku.hk \\ Paul Yang \\ Department of Mathematics \\ Princeton University \\ Princeton NJ 08544-1000 \\ yang@math.princeton.edu
}

These supporting institutions contribute to the cost of publication of this Journal, but they are not owners or publishers and have no responsibility for its contents or policies.

See inside back cover or msp.org/pjm for submission instructions.

The subscription price for 2014 is US $\$ 410 /$ year for the electronic version, and \$535/year for print and electronic.

Subscriptions, requests for back issues and changes of subscribers address should be sent to Pacific Journal of Mathematics, P.O. Box 4163, Berkeley, CA 94704-0163, U.S.A. The Pacific Journal of Mathematics is indexed by Mathematical Reviews, Zentralblatt MATH, PASCAL CNRS Index, Referativnyi Zhurnal, Current Mathematical Publications and Web of Knowledge (Science Citation Index).

The Pacific Journal of Mathematics (ISSN 0030-8730) at the University of California, c/o Department of Mathematics, 798 Evans Hall \#3840, Berkeley, CA 94720-3840, is published twelve times a year. Periodical rate postage paid at Berkeley, CA 94704, and additional mailing offices. POSTMASTER: send address changes to Pacific Journal of Mathematics, P.O. Box 4163, Berkeley, CA 94704-0163.

PJM peer review and production are managed by EditFLOW ${ }^{\circledR}$ from Mathematical Sciences Publishers.

\section{PUBLISHED BY}

\section{mathematical sciences publishers \\ nonprofit scientific publishing}

http://msp.org/

(C) 2014 Mathematical Sciences Publishers 


\section{PACIFIC JOURNAL OF MATHEMATICS}

Volume $272 \quad$ No. $2 \quad$ December 2014

Marginally trapped submanifolds in space forms with arbitrary

signature

HENRI ANCIAUX

One line complex Kleinian groups

275

Waldemar Barrera, Angel Cano and JuAn Pablo

NAVARRETE

A note on flux integrals over smooth regular domains

305

IDO BRIGHT and JOHN M. LEE

On stable commutator length in hyperelliptic mapping class groups

323

DANny Calegari, NaOyuKi Monden and Masatoshi Sato

Compositions of birational endomorphisms of the affine plane

PierRetTe CASSOU-NoguÈs and DANIEl DAigle

The Bochner formula for isometric immersions

395

ALESSANDRO SAVO

On solutions to Cournot-Nash equilibria equations on the sphere

423

MICAH WARREN

Double and triple Givental's J-functions for stable quotients invariants

439

ALEKSEY ZiNGER

Acknowledgement 